\title{
Implications of LHC search results on the $W$ boson mass prediction in the MSSM
}

\author{
S. Heinemeyer, ${ }^{a}$ W. Hollik, ${ }^{b}$ G. Weiglein ${ }^{c}$ and L. Zeune ${ }^{c}$ \\ ${ }^{a}$ Instituto de Física de Cantabria (CSIC-UC), \\ Santander, Spain \\ ${ }^{b}$ Max-Planck-Institut für Physik (Werner-Heisenberg-Institut), \\ Föhringer Ring 6, D-80805 München, Germany \\ ${ }^{c} D E S Y$, \\ Notkestraße 85, D-22607 Hamburg, Germany \\ E-mail: Sven.Heinemeyer@cern.ch, hollik@mpp.mpg.de, \\ Georg. Weiglein@desy.de, Lisa.Zeune@desy.de
}

Abstract: We present the currently most precise $W$ boson mass $\left(M_{W}\right)$ prediction in the Minimal Supersymmetric Standard Model (MSSM) and discuss how it is affected by recent results from the LHC. The evaluation includes the full one-loop result and all known higher order corrections of SM and SUSY type. We show the MSSM prediction in the $M_{W}{ }^{-} m_{t}$ plane, taking into account constraints from Higgs and SUSY searches. We point out that even if stops and sbottoms are heavy, relatively large SUSY contributions to $M_{W}$ are possible if either charginos, neutralinos or sleptons are light. In particular we analyze the effect on the $M_{W}$ prediction of the Higgs signal at about $125.6 \mathrm{GeV}$, which within the MSSM can in principle be interpreted as the light or the heavy $\mathcal{C} \mathcal{P}$-even Higgs boson. For both interpretations the predicted MSSM region for $M_{W}$ is in good agreement with the experimental measurement. We furthermore discuss the impact of possible future LHC results in the stop sector on the $M_{W}$ prediction, considering both the cases of improved limits and of the detection of a scalar top quark.

KeYWORDS: Supersymmetry Phenomenology

ARXiV EPRINT: 1311.1663 


\section{Contents}

1 Introduction 1

2 Particle sectors of the MSSM 3

3 Determination of the $W$ boson mass 5

4 Calculation of $\Delta r \quad 6$

$\begin{array}{lll}4.1 & \text { One-loop calculation in the MSSM } & 6\end{array}$

4.2 Incorporation of higher order corrections 8

5 Numerical analysis $\quad \mathbf{9}$

5.1 Prediction for the W boson mass in the SM 9

$\begin{array}{ll}5.2 \text { MSSM parameter scan: scan ranges and constraints } & 10\end{array}$

5.3 Results for the W boson mass in the MSSM 11

$\begin{array}{ll}5.4 \text { Discussion of possible future scenarios } & 19\end{array}$

6 Conclusions 21

\section{Introduction}

The recent discovery of a signal with a mass of around 125.6 GeV in the Higgs searches at ATLAS [1] and CMS [2] is compatible with the Higgs boson postulated by the Standard Model (SM), but it can also be interpreted in a variety of models of physics beyond the SM. On the other hand, the direct searches for physics beyond the SM have not resulted in a signal so far. In order to enhance the sensitivity for discriminating between different models of the underlying physics, it is useful to complement the measurements of the properties of the new state with other high-precision observables that have sensitivity to the quantum level, i.e. to loop contributions involving in principle all the particles of the considered model.

In this context, the relation between the $W$ boson mass, $M_{W}$, and the $Z$ boson mass, $M_{Z}$, in terms of the fine-structure constant, $\alpha$, the Fermi constant, $G_{\mu}$, and the parameters entering via loop contributions plays a crucial role. The accuracy of the measurement of the $W$ boson mass has significantly been improved with the latest results presented by CDF [3] and DØ [4]. Together with the results obtained at LEP [5] this gives rise to the latest world average of $[6,7]$

$$
M_{W}^{\exp }=80.385 \pm 0.015 \mathrm{GeV}
$$

i.e. to a relative experimental accuracy of better than $2 \times 10^{-4}$. Furthermore, the improved measurement of the top-quark mass, $m_{t}$, at the Tevatron and the LHC (see below for a 
discussion of the physical interpretation of those measurements) has improved the accuracy of the theoretical prediction for $M_{W}$, since the experimental error of the input parameter $m_{t}$ constitutes a dominant source of (parametric) uncertainty in the theoretical prediction, see e.g. ref. [8]. Further observables that have a high sensitivity for testing electroweak physics at the quantum level are in particular the effective leptonic weak mixing angle at the $Z$-resonance, $\sin ^{2} \theta_{\text {eff }}$, the anomalous magnetic moment of the muon, $(g-2)_{\mu}$, and rare $b$ decays such as $b \rightarrow s \gamma$. The interpretation of the constraints from $\sin ^{2} \theta_{\text {eff }}$ are complicated by the fact that the two single most precise measurements, $A_{\mathrm{LR}}$ by SLD [7] and $A_{b}^{\mathrm{FB}}$ at LEP [7], differ from each other by more than $3 \sigma$, see e.g. ref. [9] for a recent discussion. While the experimental value of $(g-2)_{\mu}$ shows a significant deviation from the SM prediction at the level of $3-4 \sigma$, which led to many interpretations in terms of new physics models (see e.g. refs. [10-12] for reviews), the analysis of rare $b$ decays so far has been inconclusive [13].

We will concentrate in the following on the prediction for the $W$ boson mass and, taking into account the latest experimental results, compare the prediction of the SM with that of its most popular extension, the Minimal Supersymmetric Standard Model (MSSM) [14-16]. Within the SM, the interpretation of the discovered new state as the SM Higgs boson implies that there is no unknown parameter anymore in the prediction for $M_{W}$. This fact considerably sharpens both the comparison with the experimental result for $M_{W}$ and with predictions in extensions of the SM such as the MSSM. Our analysis within the MSSM updates previous studies, see in particular refs. [17, 18] and references therein. Our results are based on the currently most precise prediction for $M_{W}$ in the MSSM, which we compare with the result in the SM. The MSSM prediction consists of a complete one-loop calculation for the general case of complex parameters (without flavor violation in the sfermion sector [19]), combined with all known higher-order corrections of SM and supersymmetric (SUSY) type. Compared to the result employed in ref. [17], the MSSM prediction used in the present analysis has been improved in several respects: the one-loop result in the MSSM has been reevaluated and coded in a more flexible way, which permits an improved treatment of regions of parameter space that can lead to numerical instabilities and furthermore provides the functionality to easily implement results for non-minimal SUSY models (see ref. [20] and also ref. [21] for the case of the NMSSM); the incorporation of the state-of-the-art SM result has been improved using the expressions given in ref. [22].

The top quark mass used in our evaluation corresponds to the pole mass. In our results it could easily be re-expressed in terms of a properly defined short distance mass such as the $\overline{\mathrm{MS}}$ or $\overline{\mathrm{DR}}$ mass. The parameter measured with high precision via direct reconstruction at the Tevatron and the LHC is expected to be close to the top pole mass, and we adopt this interpretation in the following. For a discussion of the systematic uncertainties arising from the difficulties how to relate the measured mass parameter to the pole mass see refs. [23, 24].

Extensive searches for SUSY particles have been performed by ATLAS and CMS. No supersymmetric particles have been detected so far in direct searches, and stringent limits were set in particular on the gluino mass and the mass of the squarks of the first 
two generations [25-28], see however refs. [29, 30]. Substantially weaker limits have been reported for the particles of the other MSSM sectors, so that third-generation squarks, stops and sbottoms, as well as the uncolored SUSY particles are significantly less constrained by LHC searches, and LEP limits still give relevant constraints [31].

In this paper we analyze the prediction for $M_{W}$ in view of the discovery of a signal in the Higgs searches at ATLAS and CMS. Within the framework of the MSSM the lighter $\mathcal{C P}$-even Higgs boson can have a mass of about $125.6 \mathrm{GeV}$ for sufficiently large $M_{A}$ and sufficiently large higher-order corrections from the scalar top sector. It is interesting to note that a mass value as high as about $125.6 \mathrm{GeV}$ for the lighter $\mathcal{C} \mathcal{P}$-even Higgs boson of the MSSM implies that $M_{A}$ has to be in the decoupling region, $M_{A} \gg M_{Z}$, which in turn has the consequence that the state at about $125.6 \mathrm{GeV}$ has a SM-like behavior, see e.g. the discussion in refs. $[32,33]$. However, also the interpretation of the discovered particle as the heavy $\mathcal{C} \mathcal{P}$-even Higgs state of the MSSM is, at least in principle, a viable possibility, see refs. [32-38]. ${ }^{1}$ We take into account the information from the mass measurement of the observed Higgs boson for these two cases, and for the light Higgs interpretation we investigate the correlation between $M_{W}$ and $\Gamma(h \rightarrow \gamma \gamma)$. The limits from Higgs searches at LEP, the Tevatron and the LHC are incorporated with the help of the code HiggsBounds (version 4.0.0) [40-42]. ${ }^{2}$ We perform scans over the relevant SUSY parameters and we analyze in detail the impact of different SUSY sectors on the prediction of $M_{W}$. We also investigate possible effects of either future limits from SUSY searches at the LHC or of the detection of a scalar top quark.

This paper is organized as follows: in the next section we give a short summary of the relevant MSSM sectors and specify our notation. In section 3 and section 4 we describe the evaluation of $M_{W}$ in the MSSM. In section 5 we present the result for $M_{W}$ from a global scan over the MSSM parameter space. We investigate the contributions from all relevant MSSM particle sectors and analyze the impact of the observed Higgs signal as well as from limits arising from searches for Higgs bosons and SUSY particles. Effects of possible future results from SUSY searches at the LHC are also discussed in this context. The conclusions can be found in section 6 .

\section{Particle sectors of the MSSM}

The prediction for $M_{W}$ in the MSSM depends on the masses, mixing angles and couplings of all MSSM particles. Sfermions, charginos, neutralinos and the MSSM Higgs bosons enter already at the one-loop level and can give substantial contributions to $M_{W}$. In this section we briefly describe the relevant MSSM sectors and fix our notation for the MSSM parameters. In our numerical analysis below we will focus on the case of real MSSM parameters. For a discussion of the possible impact of non-zero phases of the MSSM parameters see ref. [17].

\footnotetext{
${ }^{1}$ This scenario is challenged by the recent ATLAS bound on light charged Higgs bosons [39].

${ }^{2}$ The latest ATLAS results on light charged Higgs boson searches [39] are not included in this HiggsBounds version (while finalizing this paper a new HiggsBounds version including this result became available [43]).
} 
Contrary to the SM, two Higgs doublets are required in the MSSM, resulting in five physical Higgs boson degrees of freedom. At the tree level, where possible $\mathcal{C} \mathcal{P}$-violating contributions of the soft supersymmetry-breaking terms do not enter, these are the light and heavy $\mathcal{C P}$-even Higgs bosons, $h$ and $H$, the $\mathcal{C P}$-odd Higgs boson, $A$, and the charged Higgs bosons, $H^{ \pm}$. At lowest order the MSSM Higgs sector is fully described by $M_{Z}$ and two MSSM parameters, often chosen as the $\mathcal{C} \mathcal{P}$-odd Higgs boson mass, $M_{A}$, and $\tan \beta \equiv v_{2} / v_{1}$, the ratio of the two vacuum expectation values. Higher-order corrections to the Higgs boson masses can be sizeable and must be included. Particularly important are the oneand two-loop contributions from top quarks and squarks. Accordingly, the masses of the $\mathcal{C P}$-even neutral Higgs bosons and the charged Higgs boson are not free parameters (as the Higgs mass in the SM), but can be predicted in terms of the other MSSM parameters (introduced below).

The sfermion mass matrix in the gauge-eigenstate basis $\left(\tilde{f}_{L}, \tilde{f}_{R}\right)$ for one generation and flavor $f$ is given by

$$
\mathbf{M}_{\tilde{f}}=\left(\begin{array}{cc}
M_{\tilde{f}_{L}}^{2}+m_{f}^{2}+M_{Z}^{2} \cos 2 \beta\left(I_{3}^{f}-Q_{f} s_{\mathrm{w}}^{2}\right) & m_{f} X_{f} \\
m_{f} X_{f} & M_{\tilde{f}_{R}}^{2}+m_{f}^{2}+M_{Z}^{2} \cos 2 \beta Q_{f} s_{\mathrm{w}}^{2}
\end{array}\right)
$$

Here $m_{f}$ denotes the corresponding fermion mass, $I_{3}$ is the third component of the weak isospin, $Q_{f}$ the electric charge and $s_{\mathrm{w}}$ is the sine of the weak mixing angle. The $L-R$ mixing of the sfermions is determined by the off-diagonal entries

$$
m_{f} X_{f}=m_{f}\left(A_{f}-\mu\{\cot \beta, \tan \beta\}\right),
$$

where $\cot \beta$ refers to up-type sfermions and $\tan \beta$ to down-type sfermions. $A_{f}$ denotes the trilinear Higgs-sfermion coupling and $\mu$ the Higgsino mass parameter. The SUSY-breaking parameters are:

$$
\begin{aligned}
& M_{\tilde{f}_{L}}= \begin{cases}M_{\tilde{Q}_{i}} & \text { for left-handed squarks } \\
M_{\tilde{L}_{i}} & \text { for left-handed sleptons }\end{cases} \\
& M_{\tilde{f}_{R}}= \begin{cases}M_{\tilde{U}_{i}} & \text { for right-handed u-type squarks } \\
M_{\tilde{D}_{i}} & \text { for right-handed d-type squarks } \\
M_{\tilde{E}_{i}} & \text { for right-handed charged sleptons },\end{cases}
\end{aligned}
$$

where $i=1,2,3$ is the family index. Flavor violation in the sfermion sector is neglected here (see refs. $[19,44]$ for a discussion of this kind of effects in the one-loop contributions to $M_{W}$ ). The charged gauginos and Higgsinos mix with each other, yielding charginos $\tilde{\chi}_{1,2}^{ \pm}$. The corresponding mass matrix is given by

$$
\mathbf{M}_{\tilde{\chi}^{ \pm}}=\left(\begin{array}{cc}
M_{2} & \sqrt{2} M_{W} \sin \beta \\
\sqrt{2} M_{W} \cos \beta & \mu
\end{array}\right)
$$


with the soft breaking parameter $M_{2}$. The neutralinos are mixtures of the neutral gauginos and Higgsinos. The neutralino mass matrix in the basis $\left(\tilde{B}, \tilde{W}^{0}, \tilde{H}_{1}^{0}, \tilde{H}_{2}^{0}\right)$ is given by

$$
\mathbf{M}_{\tilde{\chi}^{0}}=\left(\begin{array}{cccc}
M_{1} & 0 & -M_{Z} s_{\mathrm{w}} \cos \beta & M_{Z} s_{\mathrm{w}} \sin \beta \\
0 & M_{2} & M_{Z} c_{\mathrm{w}} \cos \beta & -M_{Z} c_{\mathrm{w}} \sin \beta \\
-M_{Z} s_{\mathrm{w}} \cos \beta & M_{Z} c_{\mathrm{w}} \cos \beta & 0 & -\mu \\
M_{Z} s_{\mathrm{w}} \sin \beta & -M_{Z} c_{\mathrm{w}} \sin \beta & -\mu & 0
\end{array}\right) .
$$

The gluino is the only SUSY particle that enters only from the two-loop level onwards; thus the impact of the gluino mass, $m_{\tilde{g}}=\left|M_{3}\right|$, on the $M_{W}$ prediction is relatively small.

\section{Determination of the $W$ boson mass}

Muons decay via the weak interaction almost exclusively into $e \overline{\nu_{e}} \nu_{\mu}$ [31]. The decay was originally described within the Fermi model, which is a low-energy effective theory that emerges from the SM in the limit of vanishing momentum transfer. The Fermi constant, $G_{\mu}$, is determined with high accuracy from precise measurements of the muon life time [45] and the corresponding Fermi-model prediction including QED corrections up to $O\left(\alpha^{2}\right)$ for the point-like interaction [46-50]. Comparison of the muon-decay amplitude in the Fermi model and in the SM or extensions of it yields the relation

$$
\frac{G_{\mu}}{\sqrt{2}}=\frac{e^{2}}{8 s_{W}^{2} M_{W}^{2}}(1+\Delta r) .
$$

Here $\Delta r$ represents the sum of all contributing loop diagrams to the muon-decay amplitude after splitting off the Fermi-model type virtual QED corrections,

$$
\Delta r=\sum_{i} \Delta r_{i}
$$

with

$$
\mathcal{M}_{\text {Loop }, \mathrm{i}}=\Delta r_{i} \mathcal{M}_{\text {Born }} .
$$

This decomposition is possible since after subtracting the Fermi-model QED corrections, masses and momenta of the external fermions can be neglected, which allows to reduce all loop contributions to a term proportional to the Born matrix element, see refs. [51, 52]. By rearranging eq. (3.1), the $W$ boson mass can be calculated via

$$
M_{W}^{2}=M_{Z}^{2}\left(\frac{1}{2}+\sqrt{\frac{1}{4}-\frac{\alpha \pi}{\sqrt{2} G_{\mu} M_{Z}^{2}}(1+\Delta r)}\right) .
$$

In different models, different particles can contribute as virtual particles in the loop diagrams to the muon-decay amplitude. Therefore, the quantity $\Delta r$ depends on the specific model parameters, and eq. (3.4) provides a model-dependent prediction for the $W$ boson mass. The quantity $\Delta r$ itself does depend on $M_{W}$ as well; hence, the value of $M_{W}$ as the solution of eq. (3.4) has to be determined numerically. In practice this is done by iteration. In most cases this procedure converges quickly and only a few iterations are needed. 
In order to exploit $M_{W}$ as a precision observable providing sensitivity to quantum effects it is crucial that the theoretical predictions for $\Delta r$ are sufficiently precise with respect to the present and expected future experimental accuracies of $M_{W}$. Within the SM the full one-loop [51, 53] and two-loop [52, 54-64], as well as the leading higherorder corrections [65-73] are known. In addition a convenient fitting formula for $M_{W}$ containing all numerically relevant contributions has been developed [74], and in ref. [22] a corresponding formula for the two-loop electroweak contributions to $\Delta r$ has been given. In the MSSM the one-loop result [17, 75-85] and leading two-loop corrections have been obtained [86-89].

\section{Calculation of $\Delta r$}

Our analysis is based on a new one-loop calculation of $\Delta r$ in the MSSM with complex parameters which has been carried out using the Mathematica [90] based programs FeynArts (Version 3.5) [91-96] and FormCalc (Version 6.2) [97], see ref. [20] for further details. The one-loop result is combined with all known higher order corrections of SM and SUSY type as specified below, so that the numerical results given in this paper correspond to the currently most precise predictions for the $W$ boson mass in the SM and the MSSM.

\subsection{One-loop calculation in the MSSM}

The one-loop contributions to $\Delta r$ consist of the $W$ boson self-energy, vertex and box diagrams, and the related counter terms $(\mathrm{CT})$,

$$
\begin{aligned}
\Delta r= & W \text { Self-energy }+W \text { Self-energy CT }+ \text { Vertex }+ \text { Vertex CT }+ \text { Box } \\
= & \frac{\Sigma_{T}^{W W}(0)}{M_{W}^{2}}+\left(-\delta Z_{W}-\frac{\delta M_{W}^{2}}{M_{W}^{2}}\right)+\text { Vertex } \\
& \quad+\left(2 \delta Z_{e}-2 \frac{\delta s_{\mathrm{w}}}{s_{\mathrm{w}}}+\delta Z_{W}+\frac{1}{2}\left(\delta Z^{\mu}+\delta Z^{e}+\delta Z^{\nu_{\mu}}+\delta Z^{\nu_{e}}\right)\right)+\text { Box. }
\end{aligned}
$$

Here $\Sigma_{T}$ denotes the transverse part of a gauge boson self-energy, $\delta M_{W}$ is the counterterm for the $W$ boson mass, $\delta Z_{e}$ and $\delta s_{\mathrm{w}}$ are the renormalization constants for the electric charge and the (sine of the) weak mixing angle, respectively, while the other $\delta Z$ denote field renormalization constants. Since the $W$ boson appears only as a virtual particle, its field renormalization constant $\delta Z_{W}$ drops out in the $\Delta r$ formula. The box diagrams are themselves UV-finite in a renormalizable gauge. Choosing on-shell renormalization conditions, ${ }^{3}$ which ensures that eq. (3.1) corresponds to the relation between the physical

\footnotetext{
${ }^{3}$ The on-shell renormalization conditions correspond to the definition of the $W$ and $Z$ boson masses according to the real part of the complex pole of the propagator. This gives rise to the fact that the predictions for $\Delta r$ discussed in this paper internally make use of a definition of the gauge boson masses in terms of a Breit-Wigner shape with a fixed width. The values of the $W$ and $Z$ boson masses according to this fixed-width definition are finally converted into the running-width definition which has been adopted for the determination of the experimental values of $M_{W}$ and $M_{Z}$, see e.g. ref. [52] for further details.
} 
masses of the $W$ and $Z$ bosons, yields (neglecting the masses of the external fermions) ${ }^{4}$

$$
\begin{aligned}
\Delta r= & \frac{\Sigma_{T}^{W W}(0)-\operatorname{Re}\left(\Sigma_{T}^{W W}\left(M_{W}^{2}\right)\right)}{M_{W}^{2}}+\Pi^{A A}(0)-\frac{c_{\mathrm{w}}^{2}}{s_{\mathrm{w}}^{2}} \operatorname{Re}\left[\frac{\Sigma_{T}^{Z Z}\left(M_{Z}^{2}\right)}{M_{Z}^{2}}-\frac{\Sigma_{T}^{W W}\left(M_{W}^{2}\right)}{M_{W}^{2}}\right] \\
& +2 \frac{\operatorname{sgn} s_{\mathrm{w}}}{c_{\mathrm{w}}} \frac{\Sigma_{T}^{A Z}(0)}{M_{Z}^{2}}+\text { Vertex }+\operatorname{Box}-\frac{1}{2} \operatorname{Re}\left(\Sigma_{L}^{e}(0)+\Sigma_{L}^{\mu}(0)+\Sigma_{L}^{\nu_{e}}(0)+\Sigma_{L}^{\nu_{\mu}}(0)\right),
\end{aligned}
$$

with the photon vacuum polarization

$$
\Pi^{A A}\left(k^{2}\right)=\frac{\sum_{T}^{A A}\left(k^{2}\right)}{k^{2}} .
$$

Here $\Sigma_{L}$ denotes the left-handed part of a fermion self-energy.

The contributions to $\Delta r$ in the MSSM, besides the ones of SM type, consist of a large number of additional self-energy, vertex and box diagrams containing sfermions, (SUSY) Higgs bosons, charginos and neutralinos in the loop, see also ref. [17]. In order to determine the contribution to $\Delta r$ from a particular loop diagram, the Born amplitude has to be factored out of the one-loop muon decay amplitude, as shown in eq. (3.3). While most loop diagrams directly give a result proportional to the Born amplitude, more complicated spinor structures that do not occur in the SM case arise from box diagrams containing neutralinos and charginos. Those spinor chains can be related to the Born amplitude with the help of Fierz identities and charge conjugation relations. The reduction of the box diagrams to Born-type amplitudes leads to coefficients containing ratios of mass-squared differences of the involved particles. These coefficients can give rise to numerical instabilities in cases of mass degeneracies. In the implementation of our results (which has been carried out in a Mathematica and a Fortran version) special care has been taken of such parameter regions with mass degeneracies or possible threshold effects, so that a numerically stable evaluation is ensured.

At the one-loop level, the quantity $\Delta r$ can be split into three parts

$$
\Delta r^{(\alpha)}=\Delta \alpha-\frac{c_{\mathrm{w}}^{2}}{s_{\mathrm{w}}^{2}} \Delta \rho+\Delta r_{\mathrm{rem}}
$$

The shift of the fine structure constant $\Delta \alpha$ arises from the charge renormalization which contains the contributions from light fermions. The quantity $\Delta \rho$ contains loop corrections to the $\rho$ parameter [98], which describes the ratio between neutral and charged weak currents, and can be written as

$$
\Delta \rho=\frac{\Sigma_{T}^{Z Z}(0)}{M_{Z}^{2}}-\frac{\Sigma_{T}^{W W}(0)}{M_{W}^{2}}
$$

\footnotetext{
${ }^{4}$ We adopt here the sign conventions for the covariant derivative used in FeynArts [91-96], which are different for the SM and the MSSM. Accordingly, sgn (the sign of the term involving the SU(2) coupling in the covariant derivative) in eq. (4.2) for this choice of convention is $\operatorname{sgn}=-1$ in the $\mathrm{SM}$ and $\operatorname{sgn}=+1$ in the MSSM. eq. (4.2) agrees with the corresponding formula given in ref. [17] up to typographical errors in ref. [17].
} 
This quantity is sensitive to the mass splitting between the isospin partners in a doublet [98], which leads to a sizable effect in the SM in particular from the heavy fermion doublet. While $\Delta \alpha$ is a pure SM contribution, $\Delta \rho$ can get large contributions also from SUSY particles, in particular the superpartners of the heavy quarks. All other terms, both of SM and SUSY type, are contained in the remainder term $\Delta r_{\text {rem }}$.

\subsection{Incorporation of higher order corrections}

The one-loop result described above has been combined with all available higher-order corrections. Since the calculation of $\Delta r$ in the SM is more advanced than in the MSSM we have organized our result such that the full SM result for $\Delta r$ can be used also for the MSSM prediction of $M_{W}$. Therefore the MSSM result is split into a SM part and a SUSY part ${ }^{5}$

$$
\Delta r^{\mathrm{MSSM}}=\Delta r^{\mathrm{SM}}+\Delta r^{\mathrm{SUSY}}
$$

Writing the MSSM result in terms of eq. (4.6) ensures in particular that in the decoupling limit of the MSSM result, where all superpartners are heavy and the Higgs sector becomes SM-like, the full SM result (with $M_{H}^{\mathrm{SM}}=M_{h}$ ) is recovered, see also the discussion in ref. [17]. The SM part of $\Delta r$ up to four-loop order is given by

$$
\begin{aligned}
\Delta r^{\mathrm{SM}}=\Delta r^{(\alpha)}+\Delta r^{\left(\alpha \alpha_{s}\right)}+ & \Delta r^{\left(\alpha \alpha_{s}^{2}\right)}+\Delta r_{\text {ferm }}^{\left(\alpha^{2}\right)}+\Delta r_{\text {bos }}^{\left(\alpha^{2}\right)} \\
& +\Delta r^{\left(G_{\mu}^{2} \alpha_{s} m_{t}^{4}\right)}+\Delta r^{\left(G_{\mu}^{3} m_{t}^{6}\right)}+\Delta r^{\left(G_{\mu} m_{t}^{2} \alpha_{s}^{3}\right)}
\end{aligned}
$$

It contains, besides the one-loop contribution $\Delta r^{(\alpha)}$,

- the two-loop QCD corrections $\Delta r^{\left(\alpha \alpha_{s}\right)}[54-59]$,

- the three-loop QCD corrections $\Delta r^{\left(\alpha \alpha_{s}^{2}\right)}$ [65-68],

- the fermionic electroweak two-loop corrections $\Delta r_{\text {ferm }}^{\left(\alpha^{2}\right)}[52,60,61]$,

- the purely bosonic electroweak two-loop corrections $\Delta r_{\text {bos }}^{\left(\alpha^{2}\right)}[62-64]$,

- the mixed QCD and electroweak three-loop contributions $\Delta r^{\left(G_{\mu}^{2} \alpha_{s} m_{t}^{4}\right)}[69,72]$,

- the purely electroweak three-loop contribution $\Delta r^{\left(G_{\mu}^{3} m_{t}^{6}\right)}[69,72]$,

- and the four-loop QCD correction $\Delta r^{\left(G_{\mu} m_{t}^{2} \alpha_{s}^{3}\right)}[71]$.

The full result for the electroweak two-loop contributions in the SM involves numerical integrations of the two-loop scalar integrals, which make the corresponding code rather unwieldy and slow. Thus, we make use of the simple parametrisation that has been given in ref. [22] for the combined result of the fermionic and bosonic electroweak two-loop corrections in the SM, which approximates the exact result for $\Delta r_{\text {ferm }}^{\left(\alpha^{2}\right)}+\Delta r_{\text {bos }}^{\left(\alpha^{2}\right)}$ to better than $2.7 \times 10^{-5}$ for $10 \mathrm{GeV} \leq M_{H}^{\mathrm{SM}} \leq 1 \mathrm{TeV}$ (and the other input parameters in their $2 \sigma$

\footnotetext{
${ }^{5}$ Since the complete one-loop results for $\Delta r$ in the SM and in the MSSM are used in eq. (4.6), this splitting has an impact only from the two-loop level onwards.
} 
ranges), corresponding to an uncertainty of $0.4 \mathrm{MeV}$ for $M_{W}$. The use of a parametrisation directly for the SM prediction of $\Delta r_{\text {ferm }}^{\left(\alpha^{2}\right)}+\Delta r_{\text {bos }}^{\left(\alpha^{2}\right)}$ rather than for the full SM prediction of $M_{W}$ leads to an improved accuracy in the combination with the SUSY contributions as compared to ref. [17]. Concerning the QCD corrections, which enter from the two-loop level onwards, it should be noted that they result in a rather large (downward) shift of the $W$ boson mass prediction. It is obvious that this kind of corrections needs to be theoretically well under control in order to gain sensitivity to effects of physics beyond the SM.

The quantity $\Delta r^{\text {SUSY }}$ in eq. (4.6) denotes the difference between $\Delta r$ in the MSSM and the SM, i.e. it only involves the contributions from the additional SUSY particles and the extended Higgs sector. Beyond one-loop order, all SUSY corrections that are known to date are implemented, namely the leading reducible $\mathcal{O}\left(\alpha^{2}\right)$ two-loop corrections that can be obtained via the resummation formula given in ref. [99], the leading SUSY twoloop QCD corrections of $\mathcal{O}\left(\alpha \alpha_{s}\right)$ to $\Delta \rho$ as given in refs. [86, 87], as well as the dominant Yukawa-enhanced electroweak corrections of $\mathcal{O}\left(\alpha_{t}^{2}\right), \mathcal{O}\left(\alpha_{t} \alpha_{b}\right), \mathcal{O}\left(\alpha_{b}^{2}\right)$ to $\Delta \rho[88,89]$. In order to incorporate the latter corrections, the dominant Yukawa-enhanced electroweak corrections in the SM [100, 101] have been subtracted from the MSSM result presented in ref. [89] according to eq. (4.6). For this purpose we have identified the SM Higgs mass entering the result of refs. $[100,101]$ with the mass of the MSSM Higgs boson that has the largest coupling to gauge bosons (i.e., the MSSM Higgs boson that behaves most SM-like). In the decoupling limit, where $M_{A} \gg M_{Z}$ and all superpartners are heavy, the MSSM contribution reduces to the SM contribution with $M_{H}^{\mathrm{SM}}=M_{h}$, so that the contribution to $\Delta r^{\mathrm{SUSY}}$ vanishes as required.

\section{$5 \quad$ Numerical analysis}

Our numerical results are based on the contributions to $\Delta r$ described in the previous section (which have been implemented in a Mathematica and a Fortran version, where the latter has been used to generate the results presented below). The numerical values for the masses and effective couplings of the MSSM Higgs bosons have been evaluated with the help of the program FeynHiggs (version 2.9.4) [102-106]. We cross-checked our evaluation with the earlier results given in ref. [17] and found good agreement, at the level of about $1-2 \mathrm{MeV}$.

\subsection{Prediction for the $\mathrm{W}$ boson mass in the SM}

The mass of the signal discovered in the Higgs boson searches at the LHC about a year ago is measured mainly in the $\gamma \gamma$ and the $Z Z^{(*)}$ channels. Currently, the combined mass measurement from ATLAS is 125.5 $\pm 0.2 \pm 0.6 \mathrm{GeV}$ [107] and from CMS $125.7 \pm 0.3 \pm$ $0.3 \mathrm{GeV}$ [108]. Adding systematic and statistical errors in quadrature and determining the weighted average between the ATLAS and CMS measurements we get $M_{H}^{\mathrm{SM}}=125.64 \pm$ $0.35 \mathrm{GeV}$. Setting the SM Higgs boson mass to this value, the SM prediction for the $W$ boson mass reads (the other SM parameters have been fixed as $G_{\mu}=1.1663787 \times 10^{-5}$, $\left.M_{Z}=91.1875 \mathrm{GeV}, \alpha_{s}\left(M_{Z}\right)=0.1180, \Delta \alpha_{\text {had }}=0.02757\right)$

$$
M_{W}^{\mathrm{SM}}\left(m_{t}=173.2 \mathrm{GeV}, M_{H}^{\mathrm{SM}}=125.64 \mathrm{GeV}\right)=80.361 \mathrm{GeV} .
$$


Accordingly, the SM prediction for $M_{W}$ turns out to be below the current experimental value, $M_{W}^{\exp }=80.385 \pm 0.015 \mathrm{GeV}$, by about $1.5 \sigma$. The dominant theoretical uncertainty of the prediction for $M_{W}$ arises from the parametric uncertainty induced by the experimental error in the measurement of the top-quark mass. An experimental error of $1 \mathrm{GeV}$ on $m_{t}$ causes a parametric uncertainty on $M_{W}$ of about $6 \mathrm{MeV}$, while the parametric uncertainties induced by the current experimental error of the hadronic contribution to the shift in the fine-structure constant, $\Delta \alpha_{\text {had }}$, and by the experimental error of $M_{Z}$ amount to about $2 \mathrm{MeV}$ and $2.5 \mathrm{MeV}$, respectively. The uncertainty of the $M_{W}$ prediction caused by the experimental error of the Higgs mass $\delta M_{H}^{\exp }=0.35 \mathrm{GeV}$ is significantly smaller $(\sim 0.2 \mathrm{MeV})$. The uncertainties from unknown higher-order corrections have been estimated to be around $4 \mathrm{MeV}$ in the SM for a light Higgs boson $\left(M_{H}^{\mathrm{SM}}<300 \mathrm{GeV}\right)[74]$.

\subsection{MSSM parameter scan: scan ranges and constraints}

The prediction for $M_{W}$ in the MSSM is affected by additional theoretical uncertainties from unknown higher-order corrections of SUSY type. While in the decoupling limit those additional uncertainties vanish, they can be important if some SUSY particles, in particular in the scalar top and bottom sectors, are relatively light. The combined theoretical uncertainty from unknown higher-order corrections of SM- and SUSY-type has been estimated (for the MSSM with real parameters) in refs. [17, 89] as $\delta M_{W}=(4.7-9.4) \mathrm{MeV}$, depending on the SUSY mass scale.

In the following we will investigate the prediction for $M_{W}$ in the MSSM based on scans of the MSSM parameters over a wide range (using flat distributions). We have performed two versions of those random scans, one where the top-quark mass is kept fixed at $m_{t}=173.2 \mathrm{GeV}$ and one where also $m_{t}$ is allowed to vary in the scan. Both scans use initially $\sim 5 \times 10^{6}$ points, and dedicated smaller scans have been performed in parameter regions where the SUSY contributions to $M_{W}$ are relatively large. The scan ranges are given in Table 1 . We have assumed that the value of $M_{1}$ is fixed by the one of $M_{2}$ in terms of the usual GUT relation, $M_{1}=5 / 3 s_{\mathrm{w}}^{2} / c_{\mathrm{w}}^{2} M_{2}$. As mentioned above, we restrict our numerical analysis to the case of real parameters. We include CKM mixing, but the numerical effect turns out to be negligible (below $0.01 \mathrm{MeV}$ in $M_{W}$ ). Possible flavor violation in the SUSY sector [19] is neglected here. In order to avoid unphysical parameter regions and regions of numerical instabilities we disregard parameter points for which FeynHiggs indicates a large theoretical uncertainty in the evaluation of the Higgs mass predictions. We furthermore exclude points where stop and sbottom masses are massdegenerate within less than $0.1 \mathrm{GeV}$ causing numerical instabilities in the gluino corrections of $\mathcal{O}\left(\alpha \alpha_{s}\right)$ to $\Delta \rho$.

All MSSM points included in our results have the lightest neutralino as LSP and have SUSY particle masses that pass the lower mass limits from direct searches at LEP. The Higgs and SUSY masses are calculated from the MSSM input parameters using FeynHiggs (version 2.9.4) [103-106]. In the SM and SUSY higher-order corrections, as listed in section 4.2, the bottom-quark mass has been renormalized in the on-shell scheme. Accordingly, in our evaluation of $M_{W}$ the bottom-quark pole mass, $m_{b}^{\text {pole }}$, is used everywhere. This also applies to the calculation of the sbottom masses from the MSSM input parameters, and we 


\begin{tabular}{|ccc|}
\hline Parameter & Minimum & Maximum \\
\hline$\mu$ & -2000 & 2000 \\
$M_{\tilde{E}_{1,2,3}}=M_{\tilde{L}_{1,2,3}}$ & 100 & 2000 \\
$M_{\tilde{Q}_{1,2}}=M_{\tilde{U}_{1,2}}=M_{\tilde{D}_{1,2}}$ & 500 & 2000 \\
$M_{\tilde{Q}_{3}}$ & 100 & 2000 \\
$M_{\tilde{U}_{3}}$ & 100 & 2000 \\
$M_{\tilde{D}_{3}}$ & 100 & 2000 \\
$A_{e}=A_{\mu}=A_{\tau}$ & $-3 M_{\tilde{E}}$ & $3 M_{\tilde{E}}$ \\
$A_{u}=A_{d}=A_{c}=A_{s}$ & $-3 M_{\tilde{Q}_{12}}$ & $3 M_{\tilde{Q}_{12}}$ \\
$A_{b}$ & $-3 \max \left(M_{\tilde{Q}_{3}}, M_{\tilde{D}_{3}}\right)$ & $3 \max \left(M_{\tilde{Q}_{3}}, M_{\tilde{D}_{3}}\right)$ \\
$A_{t}$ & $-3 \max \left(M_{\tilde{Q}_{3}}, M_{\tilde{U}_{3}}\right)$ & $3 \max \left(M_{\tilde{Q}_{3}}, M_{\tilde{U}_{3}}\right)$ \\
$\tan \beta$ & 1 & 60 \\
$M_{3}$ & 500 & 2000 \\
$M_{A}$ & 90 & 1000 \\
$M_{2}$ & 100 & 1000 \\
\hline
\end{tabular}

Table 1. Parameter ranges considered in the scans. All parameters with mass dimension are given in $\mathrm{GeV}$.

have modified the corresponding routine in FeynHiggs accordingly (in the calculation of the sbottom masses furthermore a $\Delta_{b}$ [109-112] correction enters, which can be absorbed into an effective bottom-quark mass). For every parameter point we test whether it is allowed by direct Higgs searches using the code HiggsBounds (version 4.0.0) [40-42]. This code tests the compatibility of the MSSM points with the search limits from LEP, the Tevatron and the LHC. Running HiggsBounds, we take into account the theoretical uncertainties on the Higgs masses using the estimate provided by FeynHiggs.

Our results presented below improve on earlier results given in ref. [17] in several respects. We study here the impact of both the limits from the Higgs boson searches as well as from the signal observed at about $125.6 \mathrm{GeV}$. Furthermore we investigate constraints from present and possible future limits from searches for SUSY particles. On a more technical level, our analysis incorporates the SUSY two-loop corrections of $\mathcal{O}\left(\alpha_{t}^{2}\right), \mathcal{O}\left(\alpha_{t} \alpha_{b}\right)$, $\mathcal{O}\left(\alpha_{b}^{2}\right)$, which were not included in the scan results presented previously, and we perform a more detailed scan involving a larger number of sampling points.

\subsection{Results for the W boson mass in the MSSM}

In this section we study the MSSM prediction for $M_{W}$, starting in figure 1 where $M_{W}$ is displayed as a function of the top-quark mass, $m_{t}$, in the SM and the MSSM. The green area shows the MSSM parameter space that is allowed by HiggsBounds and the various other constraints described in the previous subsection. It should be noted that in this 

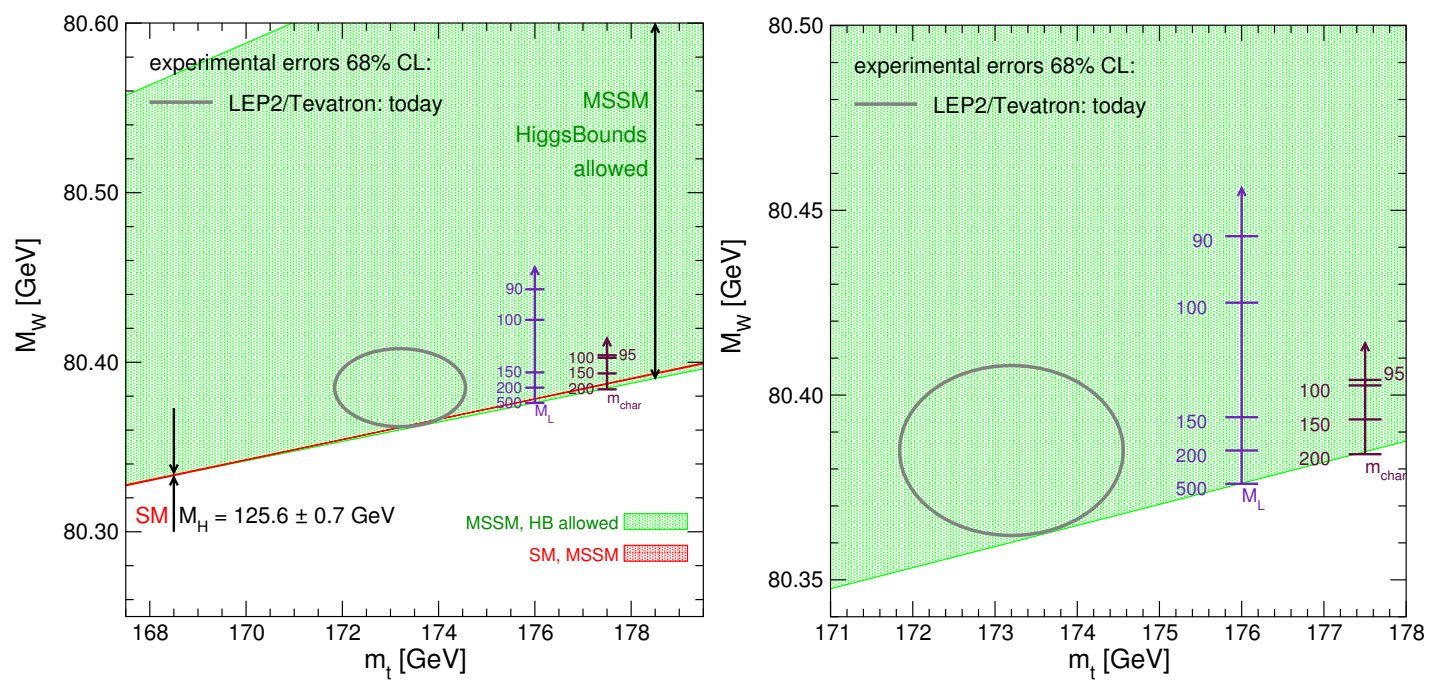

Figure 1. Prediction for $M_{W}$ as a function of $m_{t}$. Left: the green region shows the HiggsBounds allowed region for the MSSM $M_{W}$ prediction. It has been obtained by scanning over the MSSM parameters as described in the text. The cuts $m_{\tilde{t}_{2}} / m_{\tilde{t}_{1}}<2.5$ and $m_{\tilde{b}_{2}} / m_{\tilde{b}_{1}}<2.5$ are applied. The red strip indicates the overlap region of the SM and the MSSM, with $M_{H}^{S M}=125.6 \pm 0.7 \mathrm{GeV}$. The two arrows indicate the possible size of the slepton and the chargino (and neutralino) contributions. Right: zoom into the most relevant region, with the SM area omitted.

plot only the limits from the Higgs searches are considered as constraints on the MSSM parameter space, not the observed signal at about $125.6 \mathrm{GeV}$ (the latter will be discussed below). The region where the MSSM prediction for $M_{W}$ overlaps with the one in the SM is indicated by the red strip, where $M_{H}^{\mathrm{SM}}=125.6 \pm 0.7 \mathrm{GeV}$ (corresponding roughly to the $2 \sigma$ experimental error on $M_{H}$ ) has been used for the SM prediction. The left plot shows the results on a larger scale, in order to indicate the possible range of the MSSM prediction, while the right plot is a zoom into the parameter region of the MSSM near the experimental central values of $M_{W}$ and $m_{t}$. In order to obtain the MSSM prediction shown as the green band in figure 1 we have imposed as an additional restriction a limit on the mass splittings in the stop and sbottom sector, which has been implemented via the conditions $m_{\tilde{t}_{2}} / m_{\tilde{t}_{1}}<2.5$ and $m_{\tilde{b}_{2}} / m_{\tilde{b}_{1}}<2.5$. If no such condition on the mass splittings in the stop and sbottom sector were imposed, even larger values of $M_{W}$ (up to $\sim 80.8 \mathrm{GeV}$ ) would be possible in the MSSM, see also the discussion in ref. [17]. Since this parameter region far above the experimental value of $M_{W}$ is of little phenomenological interest, we will not consider it further here. While it is well-known that a non-zero SUSY contribution tends to increase the prediction for $M_{W}$ as compared to the SM case, close inspection of figure 1 reveals that there exists a small MSSM (green) region below the overlap region between the MSSM and the SM (red), which is best visible for the largest $m_{t}$ values. The reason for this feature lies in the fact that, as explained above, the SM prediction is shown for the range $M_{H}^{\mathrm{SM}}=125.6 \pm 0.7 \mathrm{GeV}$, while no restriction from the signal observed in the Higgs searches has been applied to the MSSM parameter space. As a consequence, the MSSM region (green) contains parameter points where the lightest $\mathcal{C P}$-even Higgs boson of the MSSM has a mass above the range allowed for $M_{H}^{\mathrm{SM}}$ (and below the upper bound 
on $M_{h}$ in the MSSM, which increases with increasing $m_{t}$ ). In the decoupling region, where all superpartners are heavy, the MSSM prediction for $M_{W}$ in this case corresponds to the prediction in the SM with a higher value of $M_{H}^{\mathrm{SM}}$, which yields a lower value of $M_{W} \cdot{ }^{6}$

The predictions for $M_{W}$ in the SM and the MSSM are compared with the current experimental results for $M_{W}$ and $m_{t}$ [6] which are displayed by the corresponding $68 \%$ C.L. ellipse shown in gray. One can see that the SM prediction barely touches the $68 \%$ C.L. ellipse, whereas the ellipse is fully contained in the MSSM area. It is obvious that the MSSM contains parameter regions where the MSSM prediction for $M_{W}$ is in very good agreement with the data. On the other hand, also $M_{W}$ values significantly above the experimental value are possible in the MSSM. The latter arise mainly from very light states and a large mass splitting in the stop and sbottom sector (see the discussion below).

Figure 1 shows that confronting the prediction for $M_{W}$ in the MSSM with the experimental result is of interest both for putting constraints on parameter regions that would give rise to a too high value of $M_{W}$ and for investigating the parameter region where the agreement between the MSSM prediction and the data is in fact better than for the SM case. While the deviation between the SM prediction and the experimental result for $M_{W}$ is statistically not very significant (the SM prediction is well compatible with the experimental result at the $95 \%$ C.L.), the pattern that the SM prediction is somewhat low as compared to the data has been robust for many years in spite of numerous updates of the experimental results. Focussing now on the region where we find the best agreement between the MSSM prediction for $M_{W}$ and the experimental result, it is interesting to note that in this region some of the superpartner masses are expected to be relatively light. In order to illustrate this feature we furthermore show in figure 1 the impact of the slepton sector (left arrow) and the chargino sector (right arrow), where the mass values indicated at the arrows (approximately) show the effect in $M_{W}$ arising from the contribution of a slepton and a chargino having this mass, respectively. We have chosen to display those arrows such that they start at the lower border, corresponding to the situation where all other superpartners are heavy and decoupled. For the sleptons we show the corrections to $M_{W}$ as a function of $M_{L} \equiv M_{\tilde{E}_{1,2,3}}=M_{\tilde{L}_{1,2,3}}$, where the lower limit of $\sim 90 \mathrm{GeV}$ roughly corresponds to the (fairly model-independent) limit obtained at LEP. One can see that very light sleptons, just above the LEP limit, could induce a shift in $M_{W}$ of about $60 \mathrm{MeV}$. We have checked that each generation contributes roughly the same to this effect. The major contributions to $M_{W}$ from the sleptons arise from the $\Delta \rho$ term in eq. (4.4), which is sensitive to the mass splitting between $\tilde{l}_{1,2}$ and $\tilde{\nu}_{l}$. The splitting between the sneutrinos and the sleptons becomes significant if $M_{\tilde{E}}=M_{\tilde{L}}$ and $M_{W}$ are of comparable size. The contributions to $M_{W}$ from light charginos and neutralinos are substantially smaller, but clearly not negligible in this context. They reach about $20 \mathrm{MeV}$ for $m_{\tilde{\chi}_{1}^{ \pm}} \sim 95 \mathrm{GeV}$, close to its lower mass limit from LEP. In that case, due to the assumed GUT relation between

\footnotetext{
${ }^{6}$ It should be noted that a similar kind of feature would occur even if one restricted the predicted value for $M_{h}$ in the MSSM to the same region as the range adopted for $M_{H}^{\mathrm{SM}}$. This is caused by the fact that the additional theoretical uncertainties from unknown higher-order corrections affecting the prediction for $M_{h}$ in the MSSM, which are not present in the SM where $M_{H}^{\mathrm{SM}}$ is a free input parameter, essentially lead to a broadening of the allowed range of $M_{h}$ in the MSSM as compared to $M_{H}^{\mathrm{SM}}$.
} 

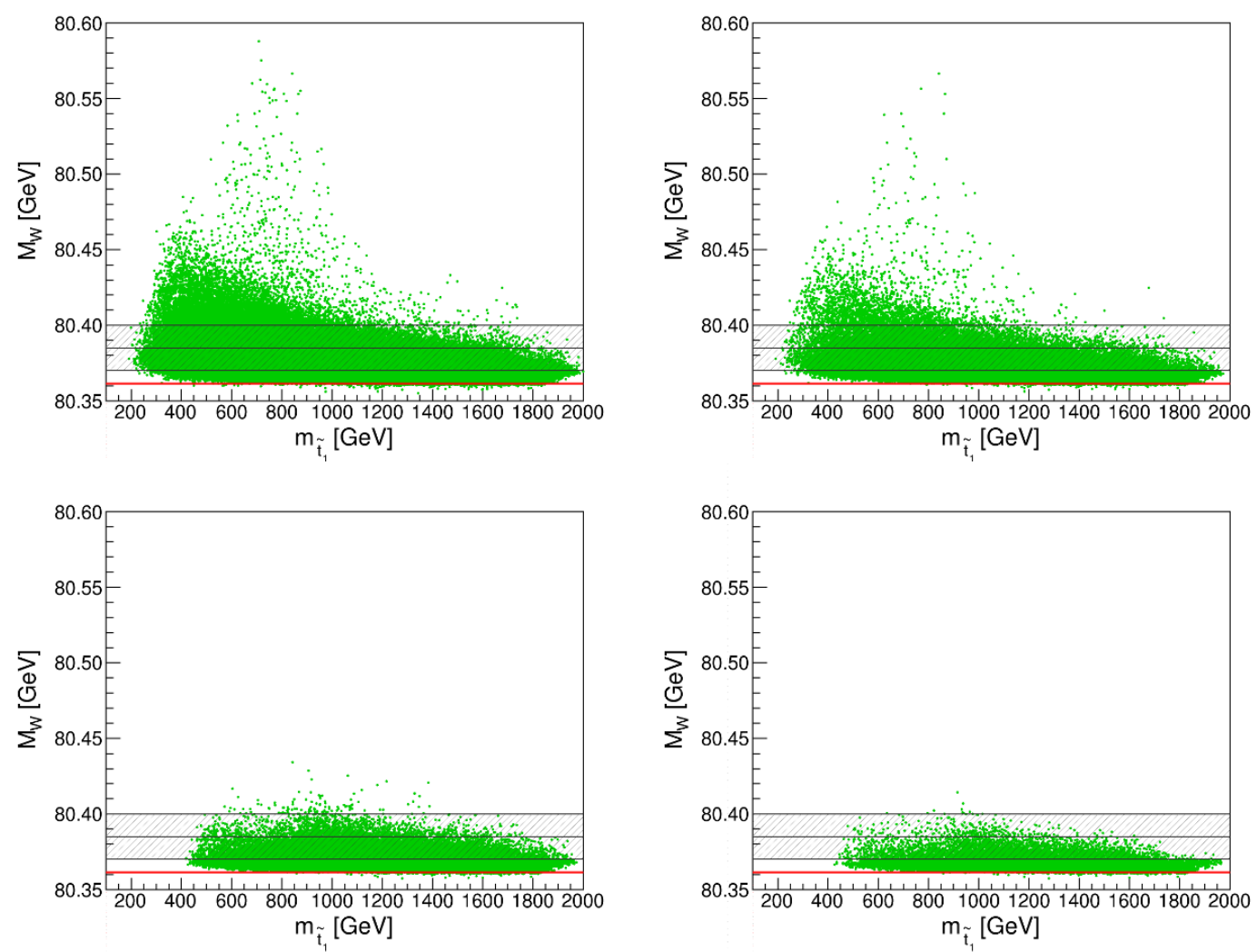

Figure 2. Prediction for $M_{W}$ as a function of the lightest stop mass $m_{\tilde{t}_{1}}$. In all plots the cuts $m_{\tilde{t}_{2}} / m_{\tilde{t}_{1}}<2.5$ and $m_{\tilde{b}_{2}} / m_{\tilde{b}_{1}}<2.5$ are applied. In the upper left plot all HiggsBounds allowed points are shown, in the upper right plot only the points are shown for which additionally the squarks of the first two generations and the gluino are heavier than $1200 \mathrm{GeV}$, in the lower left plot only the points are shown for which additionally the sbottoms are heavier than $1000 \mathrm{GeV}$, and in the lower right plot only the points are shown for which additionally also the sleptons and charginos are heavier than $500 \mathrm{GeV}$. The red line indicates the SM prediction for $M_{W}$.

$M_{1}$ and $M_{2}$, the mass of $\tilde{\chi}_{1}^{0}$ is $\sim 50 \mathrm{GeV}$. Our analysis of the contributions in the slepton and the chargino / neutralino sector shows that even if all squarks were so heavy that their contribution to the $M_{W}$ prediction were negligible, contributions from the slepton sector or the chargino / neutralino sector could nevertheless be sufficient to bring the MSSM prediction in perfect agreement with the data. This could be the case for slepton masses of about $150-200 \mathrm{GeV}$ or for a chargino mass of about $100-150 \mathrm{GeV}$. If the squark sector gives rise to a non-zero contribution to $M_{W}$ the same predicted value for $M_{W}$ could be reached with heavier sleptons and charginos / neutralinos.

In figure 2 and figure 3 we analyze in detail the dependence of $M_{W}$ on the scalar quark masses, in particular on $m_{\tilde{t}_{1}}$ and $m_{\tilde{b}_{1}}$, with $m_{t}$ fixed to $173.2 \mathrm{GeV}$. The upper left plot of figure 2 shows the prediction for $M_{W}$ (green dots) as a function of $m_{\tilde{t}_{1}}$. All points are allowed by the constraints discussed in section 5.2 and fulfill the additional constraint $m_{\tilde{t}_{2}, \tilde{b}_{2}} / m_{\tilde{t}_{1}, \tilde{b}_{1}}<2.5$. The SM prediction is shown as a red strip for $M_{H}^{\mathrm{SM}}=125.6 \pm 0.7 \mathrm{GeV}$, and the $1 \sigma$ experimental result is indicated as a gray dashed band. We checked that without 

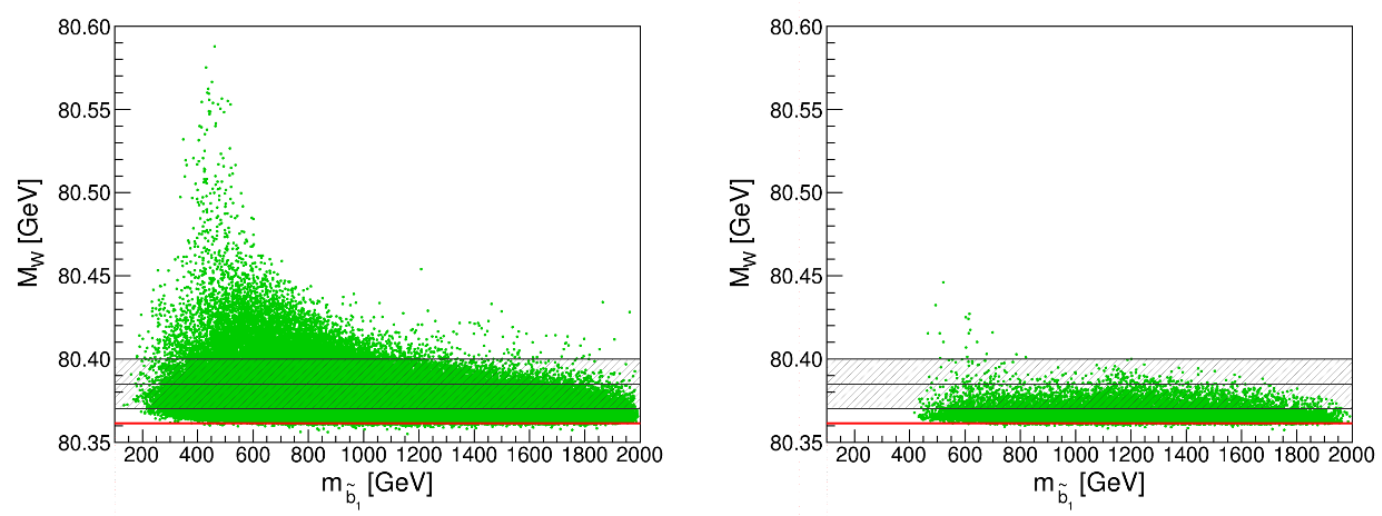

Figure 3. Prediction for $M_{W}$ as a function of the lightest sbottom mass. The cuts $m_{\tilde{t}_{2}} / m_{\tilde{t}_{1}}<2.5$ and $m_{\tilde{b}_{2}} / m_{\tilde{b}_{1}}<2.5$ are applied. In the left plot all HiggsBounds allowed points are shown, in the right plot only the points are shown for which additionally the squarks of the first two generations and the gluino are heavier than $1200 \mathrm{GeV}$, stops are heavier than $1000 \mathrm{GeV}$ and also the sleptons and charginos are heavier than $500 \mathrm{GeV}$. As above, the red line indicates the SM prediction for $M_{W}$.

the cut $m_{\tilde{t}_{2}, \tilde{b}_{2}} / m_{\tilde{t}_{1}, \tilde{b}_{1}}<2.5$ the largest $M_{W}$ values are reached for very light stop masses with a very large $(>2.5)$ splitting in the stop sector. Now the maximum of $\sim 80.6 \mathrm{GeV}$ is reached for $m_{\tilde{t}_{1}}$ around $800 \mathrm{GeV}$. The position where the maximum is reached depends strongly on the splitting between stops and sbottoms and will be further explained below (in the discussion of figure 3). In the upper right plot we only show points which have first and second generation squark masses and the gluino mass above $1.2 \mathrm{TeV}$, i.e. roughly at the limit obtained at the LHC for simplified spectra [25-28]. It can be observed that the effects on $M_{W}$ of the first and second generation squarks as well as of the gluino are rather mild. Next, in the lower left plot we only show points which in addition have $\tilde{b}$ masses above $1000 \mathrm{GeV}$ (this is a hypothetical cut that is applied for illustration purposes only; it does not reflect the current experimental situation). The fact that all MSSM points in the lower left and lower right plots have stop masses larger than $400 \mathrm{GeV}$ results from the restrictions that we have imposed, constraining the sbottom masses $(>1000 \mathrm{GeV})$ and the maximal splitting in the stop and sbottom sector $\left(m_{\tilde{t}_{2}, \tilde{b}_{2}} / m_{\tilde{t}_{1}, \tilde{b}_{1}}<2.5\right)$ at the same time. Clearly the sbottoms have a large impact on the $M_{W}$ prediction. After applying (for illustration) the sbottom mass cut the maximal $M_{W}$ values obtained in the scan are $\sim 80.43 \mathrm{GeV}$, i.e. the SUSY contributions can still be so large in this case that they can yield not only predicted $M_{W}$ values that are in good agreement with the experimental result but also ones that are significantly higher. The SUSY shift in this case is caused by the remaining contribution from the stop-sbottom sector, as well as by the contributions from charginos, neutralinos and sleptons. In order to disentangle these effects, in the lower right plot we also require (again, for illustrative purposes only) the electroweak SUSY particles to be heavy and show only points with slepton and chargino masses above $500 \mathrm{GeV}$. A direct mass limit on neutralinos is not applied. Since we fixed $M_{1} \approx \frac{1}{2} M_{2}$, all points have neutralino masses above $\sim 240 \mathrm{GeV}$. In this plot the shift in the $M_{W}$ prediction as compared to 
the SM case arises solely from the stop-sbottom sector with $m_{\tilde{b}_{1}}>1000 \mathrm{GeV}$ (neglecting the numerically insignificant contributions from the other sectors for large SUSY particle masses). One can observe that $M_{W}$ values up to the upper edge of the experimental $1 \sigma$ band $(\sim 80.400 \mathrm{GeV})$ can still be reached for $m_{\tilde{t}_{1}}$ values as high as $m_{\tilde{t}_{1}} \sim 1100 \mathrm{GeV}$ in this case. For large stop masses, $m_{\tilde{t}_{1}} \gtrsim 1100 \mathrm{GeV}$, the contributions from the stop-sbottom sector decrease as expected in the decoupling limit. ${ }^{7}$

Now we turn to figure 3 showing the $M_{W}$ prediction plotted against $m_{\tilde{b}_{1}}$. In the left plot we show all points that are allowed by HiggsBounds and the other constraints described above (in particular, $m_{\tilde{t}_{2}} / m_{\tilde{t}_{1}}<2.5$ and $m_{\tilde{b}_{2}} / m_{\tilde{b}_{1}}<2.5$ is required). In the right plot only those points are displayed for which the stops are heavier than $1000 \mathrm{GeV}$, the first and second generation squark masses as well as the gluino mass are above $1200 \mathrm{GeV}$, and the sleptons and charginos are heavier than $500 \mathrm{GeV}$. Focusing first on the left plot, one can see that it displays the same qualitative features as the upper left plot of figure 2 . While one would normally expect that the highest values for $M_{W}$ are obtained for the smallest values of $m_{\tilde{t}_{1}}$ and $m_{\tilde{b}_{1}}$, in the corresponding plots of figure 2 and figure 3 the highest $M_{W}$ values are found for $m_{\tilde{t}_{1}} \sim 800 \mathrm{GeV}$ and $m_{\tilde{b}_{1}} \sim 400 \mathrm{GeV}$. This feature is related to the imposed restriction that the maximal mass splitting for stop and sbottom masses is limited to be smaller than 2.5. The largest correction to $M_{W}$ originates from the stop-sbottom contributions to $\Delta \rho$, which depend sensitively on the mass splittings between the four squarks of the third generation. After imposing the limit on the maximal mass splittings of stops and sbottoms, these contributions become largest if the relative size of the sbottom mixing, $\left|X_{b} / \max \left(M_{\tilde{Q}_{3}}, M_{\tilde{D}_{3}}\right)\right|$, reaches its maximum. This is realized in this case for $m_{\tilde{b}_{1}} \sim 400 \mathrm{GeV}$ and $m_{\tilde{b}_{2}} / m_{\tilde{b}_{1}} \sim 2.5, m_{\tilde{t}_{1}} / m_{\tilde{b}_{1}} \sim 2$, giving rise to the maximum around $m_{\tilde{t}_{1}} \sim 800 \mathrm{GeV}$ and $m_{\tilde{b}_{1}} \sim 400 \mathrm{GeV}$ in the upper left plot of figure 2 and the left plot of figure 3 , respectively. As expected, for higher values of $m_{\tilde{b}_{1}}$ the maximum value reached for $M_{W}$ in figure 3 decreases, but $M_{W}$ values as high as the experimental central value are seen to be possible all the way up to $m_{\tilde{b}_{1}} \sim 2 \mathrm{TeV}$. In the right plot the other SUSY particles are required to be rather heavy (in particular, the stop masses are assumed to be above $1000 \mathrm{GeV}$; the other masses are restricted as described above), so that the impact of the contributions from the sbottom sector becomes apparent. While rather large contributions are possible for sbottom masses below about $800 \mathrm{GeV}$, for the highest values of $m_{\tilde{b}_{1}}$ shown in the figure the MSSM prediction for $M_{W}$ approaches the one in the SM.

So far we have only taken into account the existing limits from the Higgs searches at the LHC and other colliders (via the program HiggsBounds), but we have not explicitly imposed a constraint in view of the observed signal at $\sim 125.6 \mathrm{GeV}$. Within the MSSM (referring to the $\mathcal{C P}$-conserving case for simplicity), the signal can, at least in principle, be identified either with the light $\mathcal{C P}$-even Higgs boson $h$ or the heavy $\mathcal{C} \mathcal{P}$-even Higgs boson $H$. In figure 4 we show the SM and MSSM prediction of $M_{W}$ as a function of $m_{t}$ as obtained from our scan according to Table 1, where in the left plot the green MSSM area fulfills $M_{h}=$

\footnotetext{
${ }^{7}$ In all plots in figure 2 one can see a small gap between the MSSM points for $m_{\tilde{t}_{1}}>1900 \mathrm{GeV}$ and the SM line. This is an artefact of the chosen scan ranges: in this region the mass-splitting between $\tilde{t}_{1}$ and $\tilde{t}_{2}$ is small, and $m_{h}$ does not reach values up to $\sim 126 \mathrm{GeV}$. The $M_{W}$ value approached in the decoupling limit therefore corresponds to the SM prediction for a lower Higgs mass value.
} 

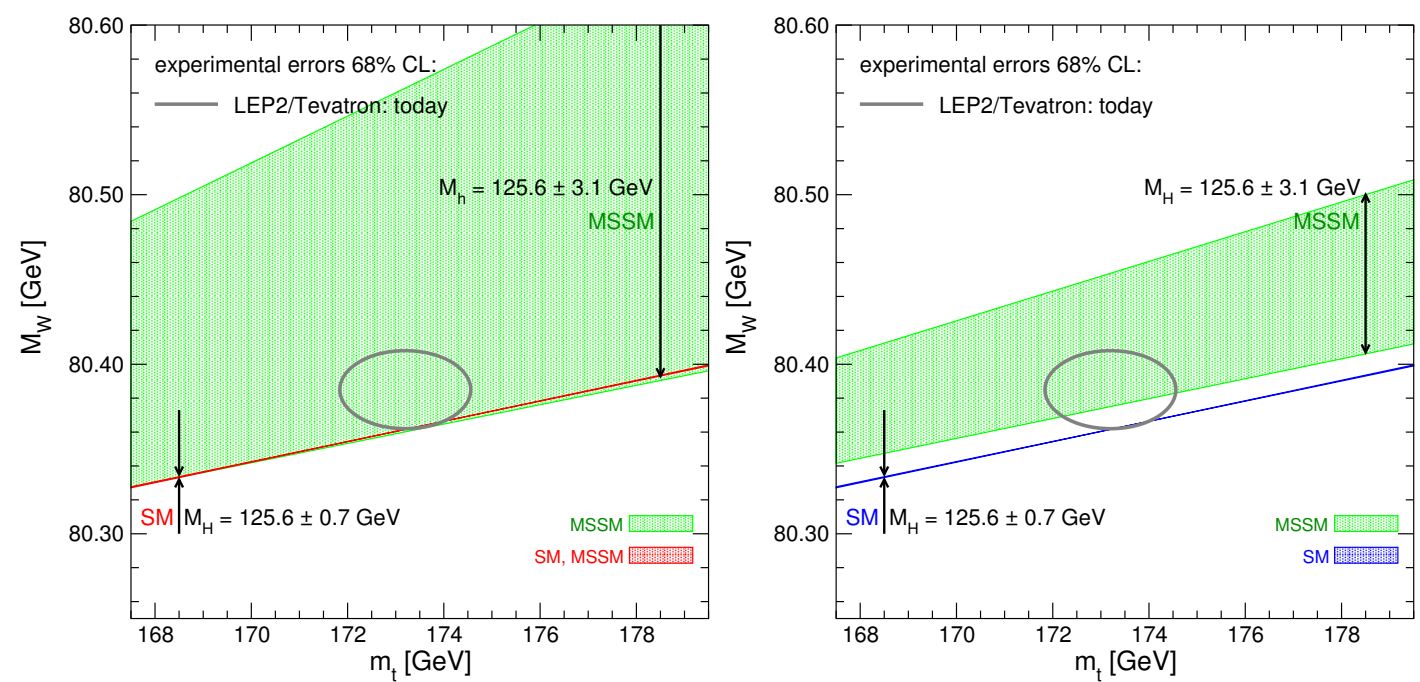

Figure 4. Prediction for $M_{W}$ as a function of $m_{t}$. The left plot shows the $M_{W}$ prediction assuming the light $\mathcal{C} \mathcal{P}$-even Higgs boson $h$ in the mass region $125.6 \pm 3.1 \mathrm{GeV}$. The red band indicates the overlap region of the SM and the MSSM with $M_{H}^{\mathrm{SM}}=125.6 \pm 0.7 \mathrm{GeV}$. The right plot shows the $M_{W}$ prediction assuming the heavy $\mathcal{C} \mathcal{P}$-even Higgs boson $H$ in the mass region $125.6 \pm 3.1 \mathrm{GeV}$. The blue band again indicates the SM region with $M_{H}^{\mathrm{SM}}=125.6 \pm 0.7 \mathrm{GeV}$. All points are allowed by HiggsBounds.

125.6 $\pm 3.1 \mathrm{GeV}$, while in the right plot the green MSSM area fulfills $M_{H}=125.6 \pm 3.1 \mathrm{GeV}$. The substantially larger uncertainty with respect to the SM experimental uncertainty of $0.7 \mathrm{GeV}$ (at the $2 \sigma$ level) arises as a consequence of the theoretical uncertainties from unknown higher-order corrections in the MSSM prediction for the Higgs boson mass. We have added a global uncertainty of $3 \mathrm{GeV}$ [104] in quadrature, yielding a total uncertainty of $3.1 \mathrm{GeV}$.

Starting with the left plot, where the light $\mathcal{C P}$-even Higgs boson has a mass that is compatible with the observed signal, we find a similar result as in figure 1 . In particular, the comparison with the experimental results for $M_{W}$ and $m_{t}$, indicated by the gray ellipse, shows a slight preference for a non-zero SUSY contribution to $M_{W}$. While the width of the MSSM area shown in green is somewhat reduced compared to figure 1 because of the additional constraint applied here (requiring $M_{h}$ to be in the range $M_{h}=125.6 \pm$ $3.1 \mathrm{GeV}$ leads to a constraint on the stop sector parameters, see, e.g., ref. [32], which in turn limits the maximal contribution to $M_{W}$ ), the qualitative features are the same as in figure 1. This is not surprising, since the limits from the Higgs searches implemented in figure 1 have already led to a restriction of the allowed mass range to the unexcluded region near the observed signal. As in figure 1 the plot shows a small MSSM region (green) below the overlap region between the MSSM and the SM (red), which is a consequence of the broadening of the allowed range of $M_{h}$ caused by the theoretical uncertainties from unknown higher-order corrections, as explained above.

In the right plot of figure 4 we show the result for the case where instead the mass of the heavy $\mathcal{C P}$-even Higgs boson is assumed to be compatible with the observed signal, i.e. $M_{H}=$ $125.6 \pm 3.1 \mathrm{GeV}$. While as mentioned above the interpretation of the discovered signal in 
terms of the heavy $\mathcal{C} \mathcal{P}$-even Higgs boson within the MSSM is challenged in particular by the recent ATLAS bound on light charged Higgs bosons [39] (which is not yet included in the version of HiggsBounds used for our analysis), ${ }^{8}$ it is nevertheless interesting to investigate to what extent the precision observable $M_{W}$ is sensitive to such a rather exotic scenario where all five states of the MSSM Higgs sector are light. The lightest $\mathcal{C P}$-even Higgs in this scenario has a heavily suppressed coupling to gauge bosons and a mass that can be significantly below the LEP limit for a SM-like Higgs, see e.g. ref. [33]. As shown in the right plot of figure 4, the constraint $M_{H}=125.6 \pm 3.1 \mathrm{GeV}$ gives rise to a situation where the MSSM region (green) does not overlap with the SM prediction (blue). This gap between the predictions of the two models is caused by the fact that $M_{H}=125.6 \pm 3.1 \mathrm{GeV}$ implies light states in the Higgs sector (in particular a light charged Higgs), which lead to a non-zero SUSY contribution to $M_{W}$ in this case, whereas for the light $\mathcal{C P}$-even Higgs boson the constraint $M_{h}=125.6 \pm 3.1 \mathrm{GeV}$ can be fulfilled in the decoupling region of the MSSM. The plot furthermore shows that the constraint $M_{H}=125.6 \pm 3.1 \mathrm{GeV}$ implies not only a lower bound on the SUSY contribution to $M_{W}$ but also a more restrictive upper bound, as can be seen from comparing the two plots in figure 4. It is interesting to note that also in the case where the heavy $\mathcal{C P}$-even Higgs is in the mass range compatible with the observed signal, the MSSM turns out to be better compatible with the experimental results for $M_{W}$ and $m_{t}$ (indicated by the gray ellipse) than the SM.

In figure 5 we analyze the dependence of the $M_{W}$ prediction on light scalar taus. In refs. $[114,115]$ it was shown that light scalar taus can enhance the decay rate of the light $\mathcal{C P}$-even Higgs boson into photons. This is of interest in view of the current experimental situation, where the signal strength in the $\gamma \gamma$ channel observed by ATLAS [116] lies significantly above the value expected in the SM (but is still compatible at the $2 \sigma$ level), while the signal strength observed in CMS [117] is currently slightly below the SM level. Since loop contributions of BSM particles to the decay width $\Gamma(h \rightarrow \gamma \gamma)$ do not have to compete with a SM-type tree-level contribution, this loop-induced quantity is of particular relevance for investigating possible deviations from the SM prediction. figure 5 shows the prediction for $M_{W}$ as a function of $\Gamma(h \rightarrow \gamma \gamma) / \Gamma(H \rightarrow \gamma \gamma)_{\mathrm{SM}}$, where the latter has been evaluated with FeynHiggs. As a starting point we use the best-fit point obtained in ref. [34] from a pMSSM-7 fit to all Higgs data (available at that time), which indeed exhibited an enhancement of $\Gamma(h \rightarrow \gamma \gamma)$ due to scalar taus with a mass close to $100 \mathrm{GeV}$. The parameters of the best fit point are $M_{A}=669 \mathrm{GeV}, \tan \beta=16.5$, $\mu=2640 \mathrm{GeV}, M_{\tilde{Q}_{3}}=M_{\tilde{U}_{3}}=M_{\tilde{D}_{3}}=1100 \mathrm{GeV}, M_{\tilde{Q}_{1,2}}=M_{\tilde{U}_{1,2}}=M_{\tilde{D}_{1,2}}=1000 \mathrm{GeV}$, $M_{\tilde{L}_{3}}=M_{\tilde{E}_{3}}=285 \mathrm{GeV}, M_{\tilde{L}_{1,2}}=M_{\tilde{E}_{1,2}}=300 \mathrm{GeV}, A_{f}=2569 \mathrm{GeV}, M_{2}=201 \mathrm{GeV}$ and $M_{3}=1000 \mathrm{GeV}$. In figure 5 the best-fit point is indicated as a black star. We vary the stau mass scale $M_{\tilde{E}_{3}}=M_{\tilde{L}_{3}}$ in the range of $280 \mathrm{GeV}$ to $500 \mathrm{GeV}$, giving rise to a corresponding variation of the lighter stau mass. The results are shown as the green line in figure 5 , where the current experimental $1 \sigma$ region for $M_{W}$ is indicated as a gray band. One can observe that for light scalar taus, corresponding to larger $\Gamma(h \rightarrow \gamma \gamma)$, the agreement of the predic-

\footnotetext{
${ }^{8}$ If the Higgs sector contains an additional singlet, as in the NMSSM, it is possible to have a SM-like second-lightest Higgs, while the charged Higgs boson can be much heavier in this case, see e.g. ref. [113].
} 


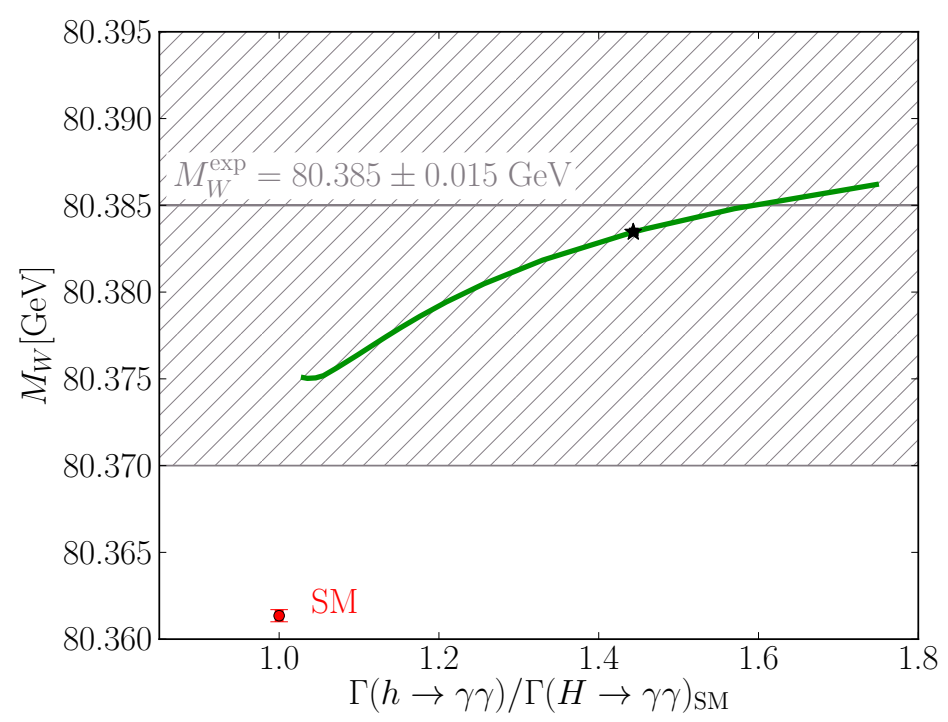

Figure 5. $M_{W}$ prediction in the MSSM as a function of $\Gamma(h \rightarrow \gamma \gamma)$, normalized to the SM value. The black star indicates the best fit point from a pMSSM-7 fit to all Higgs data (available at that time) [34]. The green line is obtained by varying $M_{\tilde{E}_{3}}=M_{\tilde{L}_{3}}$ from $280 \mathrm{GeV}$ to $500 \mathrm{GeV}$.

tion for $M_{W}$ with the experimental value is improved. A certain level of enhancement of $\Gamma(h \rightarrow \gamma \gamma)$ is also compatible with the current experimental results on the signal strength in the $\gamma \gamma$ channel. For heavy scalar taus, as obtained for $M_{\tilde{E}_{3}}=M_{\tilde{L}_{3}}=500 \mathrm{GeV}$ (and keeping the other parameters as defined above), the $M_{W}$ prediction still remains within the experimental $1 \sigma$ band, while nearly SM values for $\Gamma(h \rightarrow \gamma \gamma)$ are reached.

\subsection{Discussion of possible future scenarios}

In the final step of our investigation we discuss the precision observable $M_{W}$ in the context of possible future scenarios. We first investigate the impact of an assumed limit of $500 \mathrm{GeV}$ on stops and sbottoms (and assume that no other colored particles are observed below $1200 \mathrm{GeV}$ ). In figure 6 we show again the $M_{W}-m_{t}$ planes as presented in figure 1 (where the parameter region allowed by HiggsBounds is displayed) and in figure 4 ( $M_{h}$ or $M_{H}$ in the range of $125.6 \pm 3.1 \mathrm{GeV}$ ), but now in addition the light blue points obey the (hypothetical) mass limits for stops and sbottoms $(500 \mathrm{GeV})$ and for other colored particles $(1200 \mathrm{GeV})$. The left plot shows the HiggsBounds allowed points, whereas in the middle (right) plot $M_{h}\left(M_{H}\right)=125.6 \pm 3.1 \mathrm{GeV}$ is required. It can be observed that the light blue points corresponding to a relatively heavy colored spectrum are found at the lower end of the predicted $M_{W}$ range, i.e. in the decoupling region of the MSSM. As discussed above the largest SUSY contributions arise from the stop-sbottom sector. If lower lower mass limits on stops and sbottoms of $500 \mathrm{GeV}$ are assumed, it can be seen that the band corresponding to the possible range of predictions for $M_{W}$ in the MSSM would shrink significantly, to the region populated by the blue points. It should be noted that the prediction for $M_{W}$ in this region is in perfect agreement with the experimental measurements of $M_{W}$ and $m_{t}$. Besides the contributions of stops and sbottoms, which can still be significant even if the stops and 

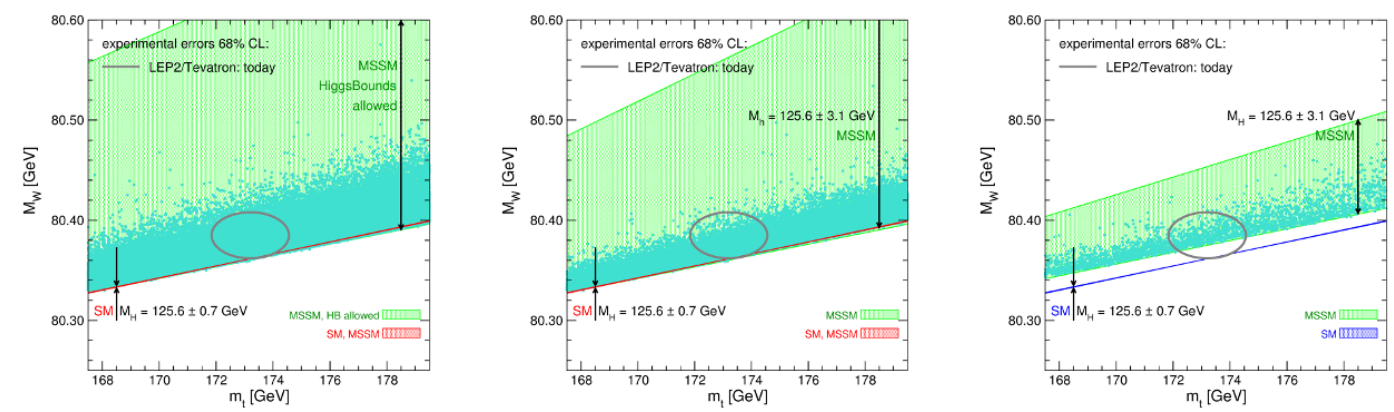

Figure 6. Prediction for $M_{W}$ as a function of $m_{t}$. The left plot shows all points allowed by HiggsBounds, the middle one requires $M_{h}$ to be in the mass region $125.6 \pm 3.1 \mathrm{GeV}$, while in the right plot $M_{H}$ is required to be in the mass region $125.6 \pm 3.1 \mathrm{GeV}$. The color coding is as in Figures 1 and 4. In addition, the blue points are the parameter points for which the stops and sbottoms are heavier than $500 \mathrm{GeV}$ and squarks of the first two generations and the gluino are heavier than $1200 \mathrm{GeV}$.

sbottoms are heavier than $500 \mathrm{GeV}$, the main SUSY corrections arise from relatively light sleptons, charginos and neutralinos, as analyzed above.

While so far we have compared the various predictions with the current experimental results for $M_{W}$ and $m_{t}$, we now discuss the impact of future improvements of these measurements. For the $W$ boson mass we assume an improvement of a factor three compared to the present case down to $\Delta M_{W}=5 \mathrm{MeV}$ from future measurements at the LHC and a prospective Linear Collider (ILC) [118], while for $m_{t}$ we adopt the anticipated ILC accuracy of $\Delta m_{t}=100 \mathrm{MeV}$ [119]. For illustration we show in figure 7 again the left plot of figure 4, assuming the mass of the light $\mathcal{C P}$-even Higgs boson $h$ in the region $125.6 \pm 3.1 \mathrm{GeV}$, but supplement the gray ellipse indicating the present experimental results for $M_{W}$ and $m_{t}$ with the future projection indicated by the red ellipse (assuming the same experimental central values). While currently the experimental results for $M_{W}$ and $m_{t}$ are compatible with the predictions of both models (with a slight preference for a non-zero SUSY contribution), the anticipated future accuracies indicated by the red ellipse would clearly provide a high sensitivity for discriminating between the models and for constraining the parameter space of BSM scenarios.

As a further hypothetical future scenario we assume that a light scalar top quark has been discovered at the LHC with a mass of $m_{\tilde{t}_{1}}=400 \pm 40 \mathrm{GeV}$, while no other new particle has been observed. As before, for this analysis we use an anticipated experimental precision of $\Delta M_{W}=5 \mathrm{MeV}$ (other uncertainties have been neglected in this analysis). Concerning the masses of the other SUSY particles, we assume lower limits of $300 \mathrm{GeV}$ on both sleptons and charginos, $500 \mathrm{GeV}$ on other scalar quarks of the third generation and of $1200 \mathrm{GeV}$ on the remaining colored particles. We have selected the points from our scan accordingly. Any additional particle observation would impose a further constraint and would thus enhance the sensitivity of the parameter determination. In figure 8 we show the parameter points from our scan that are compatible with the above constraints. All points fulfill $M_{h}=125.6 \pm 3.1 \mathrm{GeV}$ and $m_{\tilde{t}_{1}}=400 \pm 40 \mathrm{GeV}$. Yellow, red and blue 


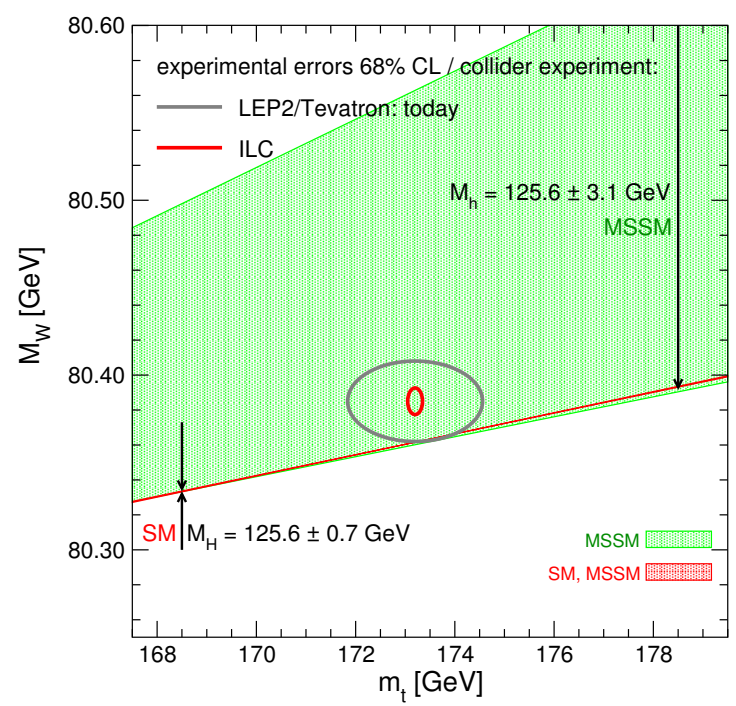

Figure 7. Prediction for $M_{W}$ as a function of $m_{t}$, as given in the left plot of figure 4 (the mass $M_{h}$ of the light $\mathcal{C} \mathcal{P}$-even Higgs boson is assumed to be in the region $125.6 \pm 3.1 \mathrm{GeV}$ ). In addition to the current experimental results for $M_{W}$ and $m_{t}$ that are displayed by the gray $68 \%$ C.L. ellipse the anticipated future precision at the ILC is indicated by the red ellipse (assuming the same experimental central values).

points have furthermore a $W$ boson mass of $M_{W}=80.375,80.385,80.395 \pm 0.005 \mathrm{GeV}$, respectively, corresponding to three hypothetical future central experimental values for $M_{W}$. The left plot in figure 8 shows the $M_{W}$ prediction as a function of the lighter sbottom mass. Assuming that the experimental central value for $M_{W}$ stays at its current value of $80.385 \mathrm{GeV}$ (red points) or goes up by $10 \mathrm{MeV}$ (blue points), the precise measurement of $M_{W}$ would set stringent upper limits of $\sim 800 \mathrm{GeV}$ (blue) or $\sim 1000 \mathrm{GeV}$ (red) on the possible mass range of the lighter sbottom. As expected, this sensitivity degrades if the experimental central value for $M_{W}$ goes down by $10 \mathrm{MeV}$ (yellow points), which would bring it closer to the SM value given in eq. (5.1). The right plot shows the results in the $m_{\tilde{b}_{1}}-m_{\tilde{t}_{2}}$ plane. It can be observed that sensitive upper bounds on those unknown particle masses could be set ${ }^{9}$ based on an experimental value of $M_{W}$ of $80.385 \pm 0.005 \mathrm{GeV}$ or $80.395 \pm 0.005 \mathrm{GeV}$ (i.e. for central values sufficiently different from the SM prediction). In this situation the precise $M_{W}$ measurement could give interesting indications regarding the search for the heavy stop and the light sbottom (or put the interpretation within the MSSM under tension).

\section{Conclusions}

We have presented the currently most precise prediction for the $W$ boson mass in the MSSM and compared it with the state-of-the-art prediction in the SM. The evaluation in the MSSM includes the full one-loop result (for the general case of complex parameters) and all known higher-order corrections of SM and SUSY type. Within the SM, interpreting the signal

\footnotetext{
${ }^{9}$ See also ref. $[120]$ for a recent analysis investigating constraints on the scalar top sector.
} 

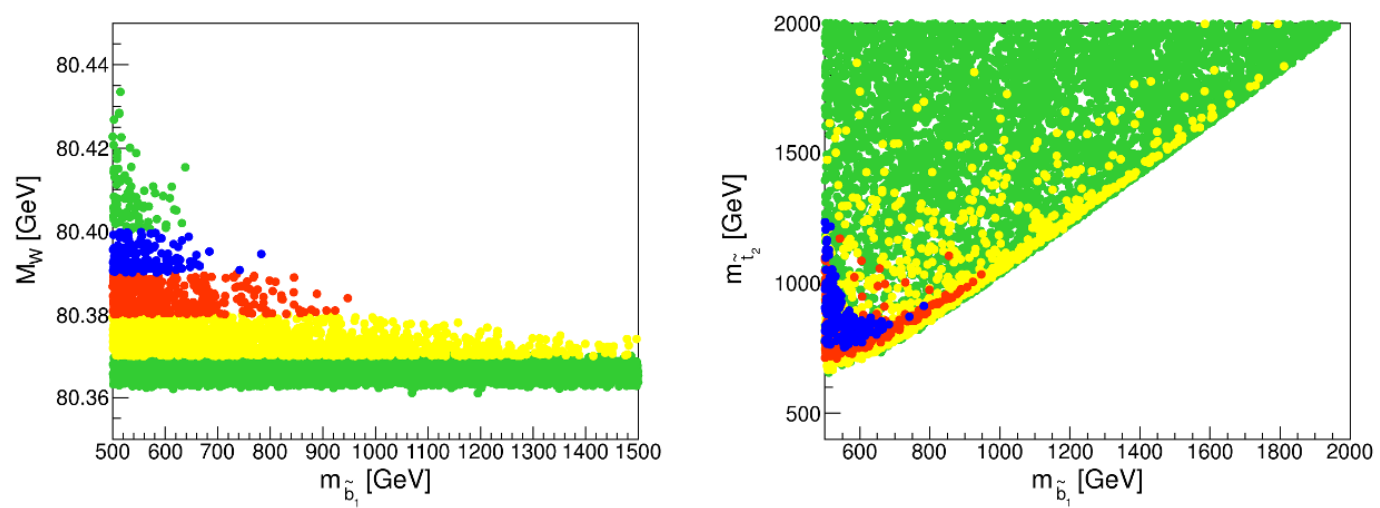

Figure 8. Results of an MSSM parameter scan illustrating the prediction for $M_{W}$ in a hypothetical future scenario assuming a measurement of $m_{\tilde{t}_{1}}=400 \pm 40 \mathrm{GeV}$ at the LHC as well as lower limits on all other SUSY particles: the assumed lower limits are $500 \mathrm{GeV}$ for the other third generation squarks, $1200 \mathrm{GeV}$ for all other colored particles, and $300 \mathrm{GeV}$ for sleptons and charginos. All displayed points fulfill $M_{h}=125.6 \pm 3.1 \mathrm{GeV}$. The yellow, red and blue points correspond to $M_{W}=$ $80.375 \pm 0.005 \mathrm{GeV}$ (yellow), $M_{W}=80.385 \pm 0.005 \mathrm{GeV}$ (red), and $M_{W}=80.395 \pm 0.005 \mathrm{GeV}$ (blue). The left plot shows the prediction for $M_{W}$ as a function of the lighter sbottom mass, $m_{\tilde{b}_{1}}$, while the right plot shows the $M_{W}$ prediction in the $m_{\tilde{b}_{1}}-m_{\tilde{t}_{2}}$ plane.

discovered at the LHC as the SM Higgs boson with $M_{H}^{\mathrm{SM}}=125.6 \mathrm{GeV}$, there is no unknown parameter in the $M_{W}$ prediction anymore. This yields $M_{W}^{\mathrm{SM}}=80.361 \mathrm{GeV}$, which is somewhat below (but compatible at the level of about $1.5 \sigma$ ) with the current experimental value of $M_{W}^{\exp }=80.385 \pm 0.015 \mathrm{GeV}$. The loop contributions from supersymmetric particles in general give rise to an upward shift in the prediction for $M_{W}$ as compared to the SM case, which tend to bring the prediction into better agreement with the experimental result. For very light superpartners of the top and bottom quarks and large mass splittings in this sector even much larger (and thus experimentally disfavored) values of $M_{W}$ are possible.

We have investigated the MSSM and SM predictions in the $M_{W}-m_{t}$ plane, updating earlier results in ref. [17] while taking into account the existing constraints from Higgs and SUSY searches. We have analyzed in this context the implications of the results of present and possible future searches for supersymmetric particles at the LHC. While the existing bounds on the gluino and the squarks of the first two generations have only a minor effect, more stringent bounds on the third generation squarks would have a drastic effect on the possible range of $M_{W}$ values in the MSSM. In particular, assuming a lower bound of $500 \mathrm{GeV}$ on the masses of the stops and sbottoms, the resulting range of predicted $M_{W}$ values in the MSSM essentially reduces to the region that is best compatible with the experimental result (corresponding to the $68 \%$ C.L. region). We have shown that MSSM predictions in exact agreement with the current experimental central value of $M_{W}$ can be reached for stop mass values as large as $m_{\tilde{t}_{1}} \sim 1.5 \mathrm{TeV}$, even if all other SUSY particles are heavy. We have furthermore pointed out that even if the squarks are so heavy that their contribution to $M_{W}$ becomes negligible, sizable SUSY contributions to $M_{W}$ are nevertheless possible if either charginos, neutralinos or sleptons are light. Analyzing the 
impact of light SUSY particles that are still allowed by LHC searches we have found that scalar leptons can give a contribution larger than $60 \mathrm{MeV}$, while light charginos can give corrections of up to $\sim 20 \mathrm{MeV}$.

Besides the impact of limits from searches for supersymmetric particles, we have analyzed the constraints arising from the Higgs signal at about $125.6 \mathrm{GeV}$. Within the MSSM this signal can be interpreted, at least in principle, either as the light or the heavy $\mathcal{C P}$ even Higgs boson (we have not addressed here the possibility of a state consisting of an admixture of $\mathcal{C P}$-even and $\mathcal{C P}$-odd components). Concerning the interpretation in terms of the light $\mathcal{C P}$-even Higgs boson, the result for $M_{W}$ turns out to be well compatible with the additional constraint that $M_{h}$ should be in the mass range compatible with the signal. The main effect of this constraint is that it somewhat reduces the allowed range of predicted $M_{W}$ values in the MSSM, improving in this way the overall compatibility with the experimental result for $M_{W}$. It is remarkable that also the rather exotic scenario where the mass of the heavy $\mathcal{C} \mathcal{P}$-even Higgs boson is required to be in the range compatible with the observed signal (which is under pressure in particular from the recent ATLAS bound on light charged Higgs bosons) leads to predicted values for $M_{W}$ that tend to be in better agreement with the experimental result than for the SM case. It is interesting to note that in this case, which corresponds to an MSSM scenario outside of the decoupling region, there is no overlap between the SM prediction and the range of MSSM predictions for $M_{W}$. A high-precision measurement of $M_{W}$ could thus yield a clear distinction between the two models in such a scenario.

As another interesting feature in the context of Higgs phenomenology, we have studied the correlation between $M_{W}$ and $\Gamma(h \rightarrow \gamma \gamma)$ via light scalar taus. Light staus contribute to the loop-induced process $h \rightarrow \gamma \gamma$, leading to an enhancement of the $\gamma \gamma$ width over the SM prediction. At the same time staus appear in the MSSM loop corrections to the muon decay, and thus light staus can also yield a sizable contribution to the prediction for $M_{W}$. We have demonstrated that light staus can have the simultaneous effect of enhancing $\Gamma(h \rightarrow \gamma \gamma)$ while bringing the $M_{W}$ prediction in perfect agreement with the current experimental central value of $M_{W}$.

As a final step we have discussed the impact of the precision observable $M_{W}$ in the context of possible future scenarios. The improved precision on $M_{W}$ and $m_{t}$ from future measurements at the LHC and in particular at a prospective Linear Collider (ILC) would significantly enhance the sensitivity to discriminate between the SM and the MSSM (as well as other BSM scenarios). Analyzing in this context the impact of possible future LHC results in the stop sector on the $M_{W}$ prediction, we have discussed a hypothetical scenario where a light stop has been detected at the LHC, while lower limits have been imposed on all other SUSY particles. We have demonstrated that, depending on the future central experimental value, a high-precision measurement of $M_{W}$ could yield quite stringent upper bounds on the mass of the heavier stop and the lighter sbottom, which could be of great interest regarding the direct searches for those particles. In case other SUSY particles were detected, this would further sharpen the sensitivity for determining unknown mass scales of the model. 


\section{Acknowledgments}

We are grateful to A. Freitas, T. Hahn, A. Kotwal, O. Stål, T. Stefaniak and D. Wackeroth for helpful discussions. This work has been supported by the Collaborative Research Center SFB676 of the DFG, "Particles, Strings and the early Universe". The work of S.H. was supported in part by CICYT (grant FPA 2010-22163-C02-01) and by the Spanish MICINN's Consolider-Ingenio 2010 Program under grant MultiDark CSD2009-00064.

Open Access. This article is distributed under the terms of the Creative Commons Attribution License which permits any use, distribution and reproduction in any medium, provided the original author(s) and source are credited.

\section{References}

[1] ATLAS collaboration, Observation of a new particle in the search for the standard model Higgs boson with the ATLAS detector at the LHC, Phys. Lett. B 716 (2012) 1 [arXiv:1207.7214] [INSPIRE].

[2] CMS collaboration, Observation of a new boson at a mass of $125 \mathrm{GeV}$ with the CMS experiment at the LHC, Phys. Lett. B $\mathbf{7 1 6}$ (2012) 30 [arXiv:1207.7235] [INSPIRE].

[3] CDF collaboration, T. Aaltonen et al., Precise measurement of the $W$-boson mass with the CDF II detector, Phys. Rev. Lett. 108 (2012) 151803 [arXiv: 1203.0275] [INSPIRE].

[4] D0 collaboration, V.M. Abazov et al., Measurement of the $W$ boson mass with the D0 detector, Phys. Rev. Lett. 108 (2012) 151804 [arXiv:1203.0293] [INSPIRE].

[5] AlePh, DELPhi, L3, OPAL, LEP Electroweak Working Group, J. Alcaraz et al., A Combination of preliminary electroweak measurements and constraints on the standard model, hep-ex/0612034 [INSPIRE].

[6] CDF, D0 collaboration, T.E.W. Group, 2012 update of the combination of CDF and D0 results for the mass of the $W$ boson, arXiv:1204.0042 [INSPIRE].

[7] AlePh, DELPHI, L3, OPAL, SLD, LEP Electroweak Working Group, SLD Electroweak Group, Sld Heavy Flavour Group, S. Schael et al., Precision electroweak measurements on the $Z$ resonance, Phys. Rept. 427 (2006) 257 [hep-ex/0509008] [INSPIRE].

[8] S. Heinemeyer, S. Kraml, W. Porod and G. Weiglein, Physics impact of a precise determination of the top quark mass at an $e^{+} e^{-}$linear collider, JHEP 09 (2003) 075 [hep-ph/0306181] [INSPIRE].

[9] S. Heinemeyer and G. Weiglein, Top, GigaZ, MegaW, arXiv:1007.5232 [InSPIRE].

[10] D. Stöckinger, The muon magnetic moment and supersymmetry, J. Phys. G 34 (2007) R45 [hep-ph/0609168] [INSPIRE].

[11] J.P. Miller, E.d. Rafael, B.L. Roberts and D. Stöckinger, Muon $(g-2)$ : experiment and theory, Ann. Rev. Nucl. Part. Sci. 62 (2012) 237 [InSPIRE].

[12] F. Jegerlehner and A. Nyffeler, The muon g-2, Phys. Rept. 477 (2009) 1 [arXiv: 0902.3360] [INSPIRE].

[13] Heavy Flavor Averaging Group, Y. Amhis et al., Averages of B-hadron, C-hadron and $\tau$-lepton properties as of early 2012, arXiv:1207.1158 [INSPIRE]. 
[14] H.P. Nilles, Supersymmetry, supergravity and particle physics, Phys. Rept. 110 (1984) 1 [INSPIRE].

[15] H.E. Haber and G.L. Kane, The search for supersymmetry: probing physics beyond the standard model, Phys. Rept. 117 (1985) 75 [INSPIRE].

[16] R. Barbieri, Looking beyond the standard model: the supersymmetric option, Riv. Nuovo Cim. 11N4 (1988) 1 [INSPIRE].

[17] S. Heinemeyer, W. Hollik, D. Stöckinger, A. Weber and G. Weiglein, Precise prediction for $M(W)$ in the MSSM, JHEP 08 (2006) 052 [hep-ph/0604147] [INSPIRE].

[18] S. Heinemeyer, W. Hollik and G. Weiglein, Electroweak precision observables in the minimal supersymmetric standard model, Phys. Rept. 425 (2006) 265 [hep-ph/0412214] [INSPIRE].

[19] S. Heinemeyer, W. Hollik, F. Merz and S. Penaranda, Electroweak precision observables in the MSSM with nonminimal flavor violation, Eur. Phys. J. C 37 (2004) 481 [hep-ph/0403228] [INSPIRE].

[20] O. Stål, G. Weiglein and L. Zeune, in preparation.

[21] F. Domingo and T. Lenz, W mass and leptonic Z-decays in the NMSSM, JHEP 07 (2011) 101 [arXiv:1101.4758] [INSPIRE].

[22] M. Awramik, M. Czakon and A. Freitas, Electroweak two-loop corrections to the effective weak mixing angle, JHEP 11 (2006) 048 [hep-ph/0608099] [INSPIRE].

[23] P.Z. Skands and D. Wicke, Non-perturbative QCD effects and the top mass at the Tevatron, Eur. Phys. J. C 52 (2007) 133 [hep-ph/0703081] [INSPIRE].

[24] A.H. Hoang and I.W. Stewart, Top mass measurements from jets and the Tevatron top-quark mass, Nucl. Phys. Proc. Suppl. 185 (2008) 220 [arXiv:0808.0222] [InSPIRE].

[25] J. Boyd, Overview of SUSY results from the ATLAS experiment, talk given at SUSY2013, August 26-31, ICTP, Trieste, Italy (2013).

[26] J. Richman, Searches for supersymmetry in the CMS experiment, talk given at SUSY2013, August 26-31, ICTP, Trieste, Italy (2013).

[27] ATLAS collaboration, https://twiki.cern.ch/twiki/bin/view/AtlasPublic/SupersymmetryPublicResults.

[28] CMS collaboration, https://twiki.cern.ch/twiki/bin/view/CMSPublic/PhysicsResultsSUS.

[29] T.J. LeCompte and S.P. Martin, Large Hadron Collider reach for supersymmetric models with compressed mass spectra, Phys. Rev. D 84 (2011) 015004 [arXiv:1105.4304] [INSPIRE].

[30] R. Mahbubani, M. Papucci, G. Perez, J.T. Ruderman and A. Weiler, Light non-degenerate squarks at the LHC, Phys. Rev. Lett. 110 (2013) 151804 [arXiv:1212.3328] [InSPIRE].

[31] Particle Data Group, J. Beringer et al., Review of particle physics, Phys. Rev. D 86 (2012) 010001 [INSPIRE].

[32] S. Heinemeyer, O. Stål and G. Weiglein, Interpreting the LHC Higgs search results in the MSSM, Phys. Lett. B 710 (2012) 201 [arXiv:1112.3026] [INSPIRE].

[33] M. Carena, S. Heinemeyer, O. Stål, C. Wagner and G. Weiglein, MSSM Higgs boson searches at the LHC: benchmark scenarios after the discovery of a Higgs-like particle, Eur. Phys. J. C 73 (2013) 2552 [arXiv:1302.7033] [INSPIRE].

[34] P. Bechtle et al., MSSM interpretations of the LHC discovery: light or heavy Higgs?, Eur. Phys. J. C 73 (2013) 2354 [arXiv:1211.1955] [InSPIRE]. 
[35] A. Bottino, N. Fornengo and S. Scopel, Phenomenology of light neutralinos in view of recent results at the CERN Large Hadron Collider, Phys. Rev. D 85 (2012) 095013 [arXiv:1112.5666] [INSPIRE].

[36] M. Drees, A supersymmetric explanation of the excess of Higgs-like events at the LHC and at LEP, Phys. Rev. D 86 (2012) 115018 [arXiv:1210.6507] [InSPIRE].

[37] K. Hagiwara, J.S. Lee and J. Nakamura, Properties of 125 GeV Higgs boson in non-decoupling MSSM scenarios, JHEP 10 (2012) 002 [arXiv:1207.0802] [INSPIRE].

[38] A. Arbey, M. Battaglia, A. Djouadi and F. Mahmoudi, The Higgs sector of the phenomenological MSSM in the light of the Higgs boson discovery, JHEP 09 (2012) 107 [arXiv: 1207.1348] [INSPIRE].

[39] ATLAS collaboration, Search for charged Higgs bosons in the $\tau+$ jets final state with $p p$ collision data recorded at $\sqrt{s}=8 \mathrm{TeV}$ with the ATLAS experiment, ATLAS-CONF-2013-090 (2013).

[40] P. Bechtle et al., Recent developments in HiggsBounds and a preview of HiggsSignals, PoS (CHARGED2012) 024 [arXiv: 1301.2345] [INSPIRE].

[41] P. Bechtle, O. Brein, S. Heinemeyer, G. Weiglein and K.E. Williams, HiggsBounds 2.0.0: confronting neutral and charged Higgs sector predictions with exclusion bounds from LEP and the Tevatron, Comput. Phys. Commun. 182 (2011) 2605 [arXiv:1102.1898] [InSPIRE].

[42] P. Bechtle, O. Brein, S. Heinemeyer, G. Weiglein and K.E. Williams, HiggsBounds: confronting arbitrary Higgs sectors with exclusion bounds from LEP and the Tevatron, Comput. Phys. Commun. 181 (2010) 138 [arXiv:0811.4169] [INSPIRE].

[43] P. Bechtle et al., HiggsBounds-4: improved tests of extended Higgs sectors against exclusion bounds from LEP, the Tevatron and the LHC, arXiv:1311.0055 [INSPIRE].

[44] M. Arana-Catania, S. Heinemeyer, M. Herrero and S. Penaranda, Higgs boson masses and B-physics constraints in non-minimal flavor violating SUSY scenarios, JHEP 05 (2012) 015 [arXiv:1109.6232] [INSPIRE].

[45] MuLan collaboration, D. Webber et al., Measurement of the positive muon lifetime and determination of the Fermi constant to part-per-million precision, Phys. Rev. Lett. 106 (2011) 041803 [arXiv: 1010.0991] [INSPIRE].

[46] R. Behrends, R. Finkelstein and A. Sirlin, Radiative corrections to decay processes, Phys. Rev. 101 (1956) 866 [INSPIRE].

[47] T. Kinoshita and A. Sirlin, Radiative corrections to Fermi interactions, Phys. Rev. 113 (1959) 1652 [INSPIRE].

[48] T. van Ritbergen and R.G. Stuart, On the precise determination of the Fermi coupling constant from the muon lifetime, Nucl. Phys. B 564 (2000) 343 [hep-ph/9904240] [INSPIRE].

[49] M. Steinhauser and T. Seidensticker, Second order corrections to the muon lifetime and the semileptonic B decay, Phys. Lett. B 467 (1999) 271 [hep-ph/9909436] [INSPIRE].

[50] A. Pak and A. Czarnecki, Mass effects in muon and semileptonic $b \rightarrow c$ decays, Phys. Rev. Lett. 100 (2008) 241807 [arXiv: 0803.0960] [INSPIRE].

[51] A. Sirlin, Radiative corrections in the $\mathrm{SU}(2) L \times \mathrm{U}(1)$ theory: a simple renormalization framework, Phys. Rev. D 22 (1980) 971 [inSPIRE].

[52] A. Freitas, W. Hollik, W. Walter and G. Weiglein, Electroweak two loop corrections to the $M(W)-M(Z)$ mass correlation in the standard model, Nucl. Phys. B 632 (2002) 189 [Erratum ibid. B 666 (2003) 305] [hep-ph/0202131] [INSPIRE]. 
[53] W. Marciano and A. Sirlin, Radiative corrections to neutrino induced neutral current phenomena in the $\mathrm{SU}(2) L \times \mathrm{U}(1)$ theory, Phys. Rev. D 22 (1980) 2695 [Erratum ibid. D 31 (1985) 213] [INSPIRE].

[54] A. Djouadi and C. Verzegnassi, Virtual very heavy top effects in LEP/SLC precision measurements, Phys. Lett. B 195 (1987) 265 [INSPIRE].

[55] A. Djouadi, $O\left(\alpha \alpha_{s}\right)$ vacuum polarization functions of the standard model gauge bosons, Nuovo Cim. A 100 (1988) 357 [inSPIRE].

[56] B.A. Kniehl, Two loop corrections to the vacuum polarizations in perturbative QCD, Nucl. Phys. B 347 (1990) 86 [inSPIRE].

[57] F. Halzen and B.A. Kniehl, $\Delta r$ beyond one loop, Nucl. Phys. B 353 (1991) 567 [InSPIRE].

[58] B.A. Kniehl and A. Sirlin, Dispersion relations for vacuum polarization functions in electroweak physics, Nucl. Phys. B 371 (1992) 141 [InSPIRE].

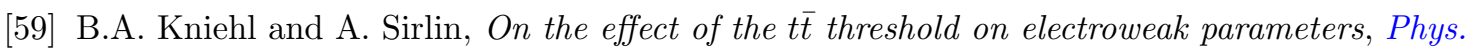
Rev. D 47 (1993) 883 [INSPIRE].

[60] A. Freitas, W. Hollik, W. Walter and G. Weiglein, Complete fermionic two loop results for the $M(W)-M(Z)$ interdependence, Phys. Lett. B 495 (2000) 338 [Erratum ibid. B 570 (2003) 260] [hep-ph/0007091] [INSPIRE].

[61] M. Awramik and M. Czakon, Complete two loop electroweak contributions to the muon lifetime in the standard model, Phys. Lett. B 568 (2003) 48 [hep-ph/0305248] [INSPIRE].

[62] M. Awramik and M. Czakon, Complete two loop bosonic contributions to the muon lifetime in the standard model, Phys. Rev. Lett. 89 (2002) 241801 [hep-ph/0208113] [INSPIRE].

[63] A. Onishchenko and O. Veretin, Two loop bosonic electroweak corrections to the muon lifetime and $M(Z)-M(W)$ interdependence, Phys. Lett. B 551 (2003) 111 [hep-ph/0209010] [inSPIRE].

[64] M. Awramik, M. Czakon, A. Onishchenko and O. Veretin, Bosonic corrections to $\Delta r$ at the two loop level, Phys. Rev. D 68 (2003) 053004 [hep-ph/0209084] [InSPIRE].

[65] L. Avdeev, J. Fleischer, S. Mikhailov and O. Tarasov, $O\left(\alpha \alpha_{S}^{2}\right)$ correction to the electroweak rho parameter, Phys. Lett. B 336 (1994) 560 [Erratum ibid. B 349 (1995) 597] [hep-ph/9406363] [INSPIRE].

[66] K. Chetyrkin, J.H. Kuhn and M. Steinhauser, Corrections of order $\mathcal{O}\left(G_{F} M_{t}^{2} \alpha_{s}^{2}\right)$ to the $\rho$ parameter, Phys. Lett. B 351 (1995) 331 [hep-ph/9502291] [INSPIRE].

[67] K. Chetyrkin, J.H. Kuhn and M. Steinhauser, QCD corrections from top quark to relations between electroweak parameters to order $\alpha_{S}^{2}$, Phys. Rev. Lett. 75 (1995) 3394 [hep-ph/9504413] [INSPIRE].

[68] K. Chetyrkin, J.H. Kuhn and M. Steinhauser, Three loop polarization function and $O\left(\alpha_{S}^{2}\right)$ corrections to the production of heavy quarks, Nucl. Phys. B 482 (1996) 213 [hep-ph/9606230] [INSPIRE].

[69] M. Faisst, J.H. Kuhn, T. Seidensticker and O. Veretin, Three loop top quark contributions to the $\rho$ parameter, Nucl. Phys. B 665 (2003) 649 [hep-ph/0302275] [INSPIRE].

[70] K. Chetyrkin, M. Faisst, J.H. Kuhn, P. Maierhofer and C. Sturm, Four-loop QCD corrections to the $\rho$ parameter, Phys. Rev. Lett. 97 (2006) 102003 [hep-ph/0605201] [INSPIRE].

[71] R. Boughezal and M. Czakon, Single scale tadpoles and $O\left(G_{F} m_{t}^{2} \alpha_{s}^{3}\right)$ corrections to the $\rho$ parameter, Nucl. Phys. B 755 (2006) 221 [hep-ph/0606232] [InSPIRE]. 
[72] J. van der Bij, K. Chetyrkin, M. Faisst, G. Jikia and T. Seidensticker, Three loop leading top mass contributions to the $\rho$ parameter, Phys. Lett. B 498 (2001) 156 [hep-ph/0011373] [INSPIRE].

[73] R. Boughezal, J. Tausk and J. van der Bij, Three-loop electroweak correction to the $\rho$ parameter in the large Higgs mass limit, Nucl. Phys. B 713 (2005) 278 [hep-ph/0410216] [INSPIRE].

[74] M. Awramik, M. Czakon, A. Freitas and G. Weiglein, Precise prediction for the $W$ boson mass in the standard model, Phys. Rev. D 69 (2004) 053006 [hep-ph/0311148] [INSPIRE].

[75] R. Barbieri and L. Maiani, Renormalization of the electroweak $\rho$ parameter from supersymmetric particles, Nucl. Phys. B 224 (1983) 32 [INSPIRE].

[76] C. Lim, T. Inami and N. Sakai, The $\rho$ parameter in supersymmetric models, Phys. Rev. D 29 (1984) 1488 [INSPIRE].

[77] E. Eliasson, Radiative corrections to electroweak interactions in supergravity GUTs, Phys. Lett. B 147 (1984) 65 [INSPIRE].

[78] Z. Hioki, One loop effects of heavy scalar quarks in supersymmetric electroweak theory, Prog. Theor. Phys. 73 (1985) 1283 [InSPIRE].

[79] J. Grifols and J. Solà, One loop renormalization of the electroweak parameters in $N=1$ supersymmetry, Nucl. Phys. B 253 (1985) 47 [inSPIRE].

[80] R. Barbieri, M. Frigeni, F. Giuliani and H. Haber, Precision measurements in electroweak physics and supersymmetry, Nucl. Phys. B 341 (1990) 309 [InSPIRE].

[81] M. Drees and K. Hagiwara, Supersymmetric contribution to the electroweak $\rho$ parameter, Phys. Rev. D 42 (1990) 1709 [InSPIRE].

[82] M. Drees, K. Hagiwara and A. Yamada, Process independent radiative corrections in the minimal supersymmetric standard model, Phys. Rev. D 45 (1992) 1725 [inSPIRE].

[83] P.H. Chankowski et al., $\Delta r$ in the MSSM, Nucl. Phys. B 417 (1994) 101 [inSPIRE].

[84] D. Garcia and J. Solà, Full one loop supersymmetric quantum effects on $M(W)$, Mod. Phys. Lett. A 9 (1994) 211 [inSPIRE].

[85] D.M. Pierce, J.A. Bagger, K.T. Matchev and R.-j. Zhang, Precision corrections in the minimal supersymmetric standard model, Nucl. Phys. B 491 (1997) 3 [hep-ph/9606211] [INSPIRE].

[86] A. Djouadi et al., Supersymmetric contributions to electroweak precision observables: QCD corrections, Phys. Rev. Lett. 78 (1997) 3626 [hep-ph/9612363] [INSPIRE].

[87] A. Djouadi et al., Leading QCD corrections to scalar quark contributions to electroweak precision observables, Phys. Rev. D 57 (1998) 4179 [hep-ph/9710438] [INSPIRE].

[88] S. Heinemeyer and G. Weiglein, Leading electroweak two loop corrections to precision observables in the MSSM, JHEP 10 (2002) 072 [hep-ph/0209305] [INSPIRE].

[89] J. Haestier, S. Heinemeyer, D. Stöckinger and G. Weiglein, Electroweak precision observables: two-loop Yukawa corrections of supersymmetric particles, JHEP 12 (2005) 027 [hep-ph/0508139] [INSPIRE].

[90] Wolfram Research Inc., Mathematica, Version 8.0, Champaign, IL U.S.A. (2010).

[91] J. Küblbeck, M. Böhm and A. Denner, Feyn Arts: Computer Algebraic Generation of Feynman Graphs and Amplitudes, Comput. Phys. Commun. 60 (1990) 165 [InSPIRE].

[92] A. Denner, H. Eck, O. Hahn and J. Küblbeck, Compact Feynman rules for Majorana fermions, Phys. Lett. B 291 (1992) 278 [INSPIRE]. 
[93] A. Denner, H. Eck, O. Hahn and J. Küblbeck, Feynman rules for fermion number violating interactions, Nucl. Phys. B 387 (1992) 467 [INSPIRE].

[94] J. Küblbeck, H. Eck, and R. Mertig, Computer algebraic generation and calculation of Feynman graphs using FeynArts and FeynCalc, Nucl. Phys. Proc. Suppl. B 29 (1992) 204.

[95] T. Hahn, Generating Feynman diagrams and amplitudes with FeynArts 3, Comput. Phys. Commun. 140 (2001) 418 [hep-ph/0012260] [INSPIRE].

[96] T. Hahn and C. Schappacher, The implementation of the minimal supersymmetric standard model in FeynArts and FormCalc, Comput. Phys. Commun. 143 (2002) 54 [hep-ph/0105349] [INSPIRE].

[97] T. Hahn and M. Pérez-Victoria, Automatized one loop calculations in four-dimensions and D-dimensions, Comput. Phys. Commun. 118 (1999) 153 [hep-ph/9807565] [INSPIRE].

[98] M. Veltman, Limit on mass differences in the Weinberg model, Nucl. Phys. B 123 (1977) 89 [INSPIRE].

[99] M. Consoli, W. Hollik and F. Jegerlehner, The effect of the top quark on the $M(W)-M(Z)$ interdependence and possible decoupling of heavy fermions from low-energy physics, Phys. Lett. B 227 (1989) 167 [INSPIRE].

[100] R. Barbieri, M. Beccaria, P. Ciafaloni, G. Curci and A. Vicere, Two loop heavy top effects in the standard model, Nucl. Phys. B 409 (1993) 105 [INSPIRE].

[101] J. Fleischer, O. Tarasov and F. Jegerlehner, Two loop heavy top corrections to the $\rho$ parameter: a simple formula valid for arbitrary Higgs mass, Phys. Lett. B 319 (1993) 249 [INSPIRE].

[102] T. Hahn, S. Heinemeyer, W. Hollik, H. Rzehak and G. Weiglein, FeynHiggs: a program for the calculation of MSSM Higgs-boson observables - Version 2.6.5, Comput. Phys. Commun. 180 (2009) 1426 [inSPIRE].

[103] M. Frank et al., The Higgs boson masses and mixings of the complex MSSM in the Feynman-diagrammatic approach, JHEP 02 (2007) 047 [hep-ph/0611326] [INSPIRE].

[104] G. Degrassi, S. Heinemeyer, W. Hollik, P. Slavich and G. Weiglein, Towards high precision predictions for the MSSM Higgs sector, Eur. Phys. J. C 28 (2003) 133 [hep-ph/0212020] [INSPIRE].

[105] S. Heinemeyer, W. Hollik and G. Weiglein, The masses of the neutral CP-even Higgs bosons in the MSSM: accurate analysis at the two loop level, Eur. Phys. J. C 9 (1999) 343 [hep-ph/9812472] [INSPIRE].

[106] S. Heinemeyer, W. Hollik and G. Weiglein, FeynHiggs: a program for the calculation of the masses of the neutral CP even Higgs bosons in the MSSM, Comput. Phys. Commun. 124 (2000) 76 [hep-ph/9812320] [INSPIRE].

[107] ATLAS collaboration, Combined measurements of the mass and signal strength of the Higgs-like boson with the ATLAS detector using up to $25 \mathrm{fb}^{-1}$ of proton-proton collision data, ATLAS-CONF-2013-014 (2013).

[108] CMS collaboration, Combination of standard model Higgs boson searches and measurements of the properties of the new boson with a mass near $125 \mathrm{GeV}$, CMS-PAS-HIG-13-005 (2013).

[109] R. Hempfling, Yukawa coupling unification with supersymmetric threshold corrections, Phys. Rev. D 49 (1994) 6168 [INSPIRE].

[110] L.J. Hall, R. Rattazzi and U. Sarid, The top quark mass in supersymmetric $\mathrm{SO}(10)$ unification, Phys. Rev. D 50 (1994) 7048 [hep-ph/9306309] [INSPIRE]. 
[111] M.S. Carena, M. Olechowski, S. Pokorski and C. Wagner, Electroweak symmetry breaking and bottom-top Yukawa unification, Nucl. Phys. B 426 (1994) 269 [hep-ph/9402253] [INSPIRE].

[112] M.S. Carena, D. Garcia, U. Nierste and C.E. Wagner, Effective lagrangian for the $\bar{t} b H^{+}$ interaction in the MSSM and charged Higgs phenomenology, Nucl. Phys. B 577 (2000) 88 [hep-ph/9912516] [INSPIRE].

[113] O. Stål and G. Weiglein, Light NMSSM Higgs bosons in SUSY cascade decays at the LHC, JHEP 01 (2012) 071 [arXiv:1108.0595] [InSPIRE].

[114] M. Carena, S. Gori, N.R. Shah, C.E. Wagner and L.-T. Wang, Light stau phenomenology and the Higgs $\gamma \gamma$ rate, JHEP 07 (2012) 175 [arXiv: 1205.5842] [INSPIRE].

[115] M. Carena, S. Gori, N.R. Shah and C.E. Wagner, A 125 GeV SM-like Higgs in the MSSM and the $\gamma \gamma$ rate, JHEP 03 (2012) 014 [arXiv:1112.3336] [INSPIRE].

[116] ATLAS collaboration, Measurements of the properties of the Higgs-like boson in the two photon decay channel with the ATLAS detector using $25 \mathrm{fb}^{-1}$ of proton-proton collision data, ATLAS-CONF-2013-012 (2013).

[117] CMS collabroation, Updated measurements of the Higgs boson at $125 \mathrm{GeV}$ in the two photon decay channel, CMS-PAS-HIG-13-001 (2013).

[118] M. Baak et al., Study of electroweak interactions at the energy frontier, arXiv:1310.6708 [INSPIRE].

[119] H. Baer et al., The International Linear Collider technical design report - Volume 2: physics, arXiv: 1306.6352 [INSPIRE].

[120] V. Barger, P. Huang, M. Ishida and W.-Y. Keung, Scalar-top masses from SUSY loops with $125 \mathrm{GeV}$ mh and precise Mw, Phys. Lett. B 718 (2013) 1024 [arXiv:1206.1777] [inSPIRE]. 